(C) 2019 IEEE. Personal use of this material is permitted. Permission from IEEE must be obtained for all other uses, in any current or future media, including reprinting/republishing this material for advertising or promotional purposes, creating new collective works, for resale or redistribution to servers or lists, or reuse of any copyrighted component of this work in other works.

\title{
A Hierarchical Approach for Dynamic Fault Trees Solution through Semi-Markov Process
}

\author{
Koorosh Aslansefat, Member, IEEE, and Gholam-Reza Latif-Shabgahi, Member, IEEE
}

\begin{abstract}
Dynamic Fault Tree (DFT) is a top-down deductive technique extended to model systems with complex failure behaviours and interactions. In two last decades, different methods have been applied to improve its capabilities such as computational complexity reduction, modularization, intricate failure distribution and reconfiguration. This paper uses SemiMarkov Process theorem for DFT solution with the motivation of obviating the model state-explosion, considering non-exponential failure distribution through a hierarchical solution. In addition, in the proposed method, a universal semi-Markov process for static and dynamic gates is introduced that can generalize dynamic behaviours like functional dependencies, sequences, priorities and spares in a single model. The efficiency of the method regarding precision and competitiveness with commercial tools, repeated events consideration, computational complexity reduction, nonexponential failure distribution consideration and repairable events in DFT is studied by a number of examples, and the results are then compared to those of the selected existing methods.
\end{abstract}

Index Terms - Dynamic fault tree, functional dependency, hierarchical solution, reliability, semi-Markov model.

\section{ACRONYMS}

$\begin{array}{ll}\text { CDF } & \text { Cumulative Distribution Function } \\ \text { CTMC } & \text { Continuous-Time Markov Chain } \\ \text { CSP } & \text { Cold Standby Spare } \\ \text { DFT } & \text { Dynamic Fault Tree } \\ \text { FDEP } & \text { Functional DEPendence } \\ \text { HCAS } & \text { Hypothetical Cardiac Assist System } \\ \text { HSP } & \text { Hot Standby Spare } \\ \text { LST } & \text { Laplace Stieltjes Transform } \\ \text { LSH } & \text { Load Sharing } \\ \text { PAND } & \text { Priority AND } \\ \text { SMP } & \text { Semi-Markov Process } \\ \text { WSP } & \text { Warm Standby Spare }\end{array}$

NOTATION

$\alpha \quad$ Dormancy Factor

$\lambda \quad$ Failure Rate (FR)

$\lambda_{i} \quad$ Failure Rate of Component $i$

$L^{-1}\{\} \quad$ The inverse of Laplace Stieltjes Transformation

$L\{\} \quad$ Laplace Stieltjes Transformation

K. Aslansefat is with the Computer Department, University of Hull, Hull, UK. Postal Code: HU6 7SR (e-mail: k.aslansefat-2018@hull.ac.uk ).

$\begin{array}{ll}\gamma & \text { Non-SEQ Factor } \\ \beta & \text { Non-PAND Factor } \\ F(t) & \text { Probability Distribution Function in Time Domain } \\ f(t) & \text { Probability Density Function in Time Domain } \\ f^{*}(s) & \text { Probability Distribution Function in LST Domain } \\ C_{d} & \text { The probability of switch work correctly } \\ P_{i} & \text { The probability of State } i^{\text {th }} \text { in (Semi) Markov Model } \\ P & \text { Probability Vector of SMP (in LST form) } \\ R(t) & \text { Reliability of a system at any time } t \\ \mu & \text { Repair Rate } \\ \mu_{i} & \text { Repair Rate of Component } i \\ G(t) & \text { Sojourn time Matrix in SMP } \\ \tilde{g}(t) & \text { Sojourn time Matrix in SMP (in LST form) } \\ S & \text { States vector of CTMC } \\ S_{i} & \text { State } i^{\text {th }} \text { in CTMC } \\ Q(t) & \text { Expression: Unreliability of a system at any time } t \\ \tilde{q}(s) & \text { Matrix: Transient Matrix in SMP }\end{array}$

\section{INTRODUCTION}

$\mathrm{F}$ fault-tolerant systems such as toxic and hazardous chemical processes, traffic control, railways and aviation systems, medical and surgery equipment need to be designed with high reliability. Reliability is the probability of a system completing its expected function without any failure during its mission time [1]. In other words, it guarantees human life, environmental health, and financial assurance. The accurate evaluation of reliability is one of the challenging engineering areas. three main methods and theories are applied for reliability evaluation; i) state-space methods such as Continuous-Time Markov Chain (CTMC), Semi-Markov Process (SMP) and Markov Regenerative Process (MRGP) [2], ii) numerical methods like Monte Carlo and probabilistic expressions $[3,4,5]$, and iii) combinatorial methods such as Reliability Block Diagram (RBD) and static Fault Tree (FT) [6].

G. R. Latif-Shabgahi, was with Electronic and Computer Department, Abbaspur Campus, Shahid Beheshti University, Tehran, Iran, (e-mail: gh latif@sbu.ac.ir ). 
Static FT is a top-down graphical deductive technique, which is powerful in the description of systems' failures and their interactions. In addition, static FT has an independent reliability solution. However, the weakness of the static FT is in its inability to model the dynamic behavior of systems such as functional and sequence dependence, spare and backup systems, priority and repair. Dynamic Fault Tree (DFT) is introduced to model dynamic and time-dependent behavior of systems through the novel gates introduced. Similar to static FT, DFT is powerful in the graphical representation of the system's failure interactions..

This paper has two main individual motivations; I) Proposing a hierarchical approach that can approximately evaluate the reliability of DFTs based on SMP theorem and without state explosion, and II) Introducing a universal five-state SMP in which different static and dynamic gates can be defined at once. In addition, thanks to the SMP theory dealing with nonexponential failure distributions and complex basic events [7, 8,9 ] can be considered as a secondary objective of the paper.

The paper presents a solution for the problems of DFTs' problems and introduces a novel SMP-based hierarchical method to solve DFTs with i) fewer states and state-transition explosions, ii) non-exponential failure distribution and iii) analytical results. Furthermore, a novel universal SMP model is introduced to different model types of static and dynamic gates, such as AND, OR, SEQ, FDEP, PAND, SPARE, and Load Sharing. In this model, the probability of switch functioning is considered to make a difference between CSP and SEQ, HSP and AND. Generally, the proposed approach is an approximate solution that can solve the DFT hierarchically limited regarding the existing dependencies, repeated events or repairs. However, after separating dependent modules, 1) In the proposed method, the output of SEQ, OR, and POR gates with any complexity in their inputs will be precise. In addition, in the AND and PAND gates when a complex event or a sub-tree is connected to the first input and a simple event connected to the second input, the output will be precise. Otherwise, the output will be approximate for other gates and other situations for AND and PAND gates the output will be approximate. SMP gives both numerical and analytical solutions. The proposed method uses the Laplace Stieltjes Transform (LST) to create analytical results which enable the user to compute other system parameters such as sensitivity, MTTF, etc.

The organization of this paper is as follows. In section II, a brief literature on the CMTC-based solutions of DFTs is studied. Section III describes the SMP analytical solutions by an example of dynamic gates and presents a universal semiMarkov model of static and dynamic gates for the first time. Section IV presents the proposed method of this paper based on SMP theorem and general equations. Section V provides a number of examples and compares the results with other research works. Section VI discusses the capability and limitation of the proposed method briefly. The paper terminates with some concluding remarks and future works.

\section{LITERATURE ON CTMC BASED RELIABILITY SOLUTIONS OF DFTS}

The dynamic nature of DFT makes solving this kind of FT more difficult than static ones. Therefore, a number of research works are conducted on different solution methods for reliability evaluation of DFT. The main idea behind the solving DFT was using CMTC, but later, diagram-based methods such as Decision Diagrams (BDD, SBDD, OBDD and MDD) [10], algebraic methods [11, 12, 13], Petri net [14], Interactive Markov chains [15] and Monte Carlo simulation [16] are presented for reliability solution of DFT's each one of which has its own advantages and disadvantages.

For the first time reference [21] introduced the concept of dynamic gates and dynamic fault trees in 1991. This tree was being solved through CTMC. The reference also suggested a systematic way for conversion of DFT to its CTMC equivalent. Following this, reference [22] evaluated the behavior of systems with imperfect coverage in 1993. The conversion of DFT into its equivalent CTMC as well as automatic inserting of imperfect coverage into CTMC is also studied in this reference. Two benchmarks named Fault Tolerant Parallel Processors (FTPP) and Mission Avoidance Systems (MAS) that used, later, by many researchers, were also introduced in this article. The reliability analysis of DFT in the presents of transient and permanent faults, failure dependencies, recovery of system and reconfiguration of FTPP benchmark were studied in [22]. In 1995, reference [23] presented two algorithms for reliability analysis of fault tolerant systems with imperfect coverage. The first algorithm used CTMC and the second used BDD, it was shown that the BDD-based algorithm decreases significantly

In 1999, reference [24] discussed the validity of methods introduced in previous research works and suggested a systematic way for improving the conversion procedure accuracy. This was the first reference that presented the CTMC model of PAND, FDEP and SPARE gates, cascaded FDEP and shared spares.

In 2001, reference [25] analyzed and categorized methods for describing uncertainty of the model regarding reliability bounds, confidence intervals and probability distribution. Furthermore, using analytic method based on CTMC, uncertainty in AND gate and an example for two parallel bus are analyzed by considering imperfect coverage and repair. Monte Carlo simulation method is used besides analytical method for considering uncertainty in this paper and a comparison between these two methods are then presented. The final results achieved from this paper include the proximity of the answers with considering uncertainty in comparison with the answers without considering uncertainty to reality. In 2002, NASA prepared a report on FT and its usage in NASA's applications and also regarding DFT and converting it into CTMC. It made use of regarding imperfect coverage in DFT, etc. [26].

In 2007, reference [27] presented a scheme for decomposing DFT into independent modules and solving them by the use of CTMC and BDD. Besides, it presented a software named Dynamic Fault Tree Analyzer (DyFA). Among the benefits of the presented method, we can refer to accuracy and less time 
consumption in DFT's solutions. Reference [28] is a thesis conducted on presenting a new method for evaluating performability and reliability of embedded and fault tolerant systems in airplanes and cars. It uses CTMC for modelling and considering dynamic behavior of the system in fault tree. Among the innovations of this research, one can refer to modeling components' failure by CTMC as basic event, besides regarding associated failure rates among components. Reference [29] dealt with a method for computing the probability of top event in DFT with PAND gate and with repeated basic events. In this paper, it is assumed that the basic events occur independently with exponential distribution and the component whose failure corresponds to the occurrence of the basic event is non-repairable. This proposed method obtains the occurrence probability of the output event in a PAND gate by CTMC. Then, the top event probability is given by a cut set approach and the inclusion-exclusion formula. An efficient procedure to obtain the probabilities corresponding to logical products in the inclusion-exclusion formula is presented. Moreover, it compares its achieved results with results obtained from Galileo and validates its method.

Reference [37] provides the subjects which were presented in a thesis [28] in an article and evaluated reliability of reinforce circuit for Power Factor Correction (PFC) using CTMC and fault tree. In 2012, reference [38] proposed a fuzzy-based method for reliability evaluation with uncertainty in fuzzy dynamic fault tree (FDFT). It makes use of fuzzy CTMC for solving fuzzy dynamic fault tree. This study is employed on a practical example of automatic hydraulic system cutting machine (CNC). An important point that should be noted in this study is that in the given dynamic fault tree example, only FDEP gate exists and fuzzy evaluation of other gates are left vague. They also use their method in another paper for evaluating driver in array of solar cells [39]. A year later, reference [40] statistically evaluated dynamic fault tree with PAND gate in which it proposed a method for converting PAND gate into AND gate along with considering some dependent conditional events. Moreover, in this study, an AND gate called CAND gate which is assumed to be dependent upon conditional events is introduced. In this study, CTMC for PAND and CAND gates are provided along with a description of their differences. Finally, this paper validates its method on FTPP's benchmark.

In 2014, reference [41] presented a chapter of his book as evaluating reliability using enhanced DFT in which it divided solving dynamic fault tree into two sections. It solves static section by using BDD and dynamic section by using CTMC. The other type of CMTC exists which is called "Input/Output Interactive CTMC (IMC)" and that is used for DFT solutions. Because of limitation in number of pages, this paper does not study I/O IMCs. (Refer to [42, 43, 44, 45, 46, 15, 47, 48]).

In 2015, regarding the Dynamic Fault Tree based reliability modelling and evaluation, more than one hundred and fifty papers, standards and tools has been reviewed by [49]. The paper has studied different extensions such as repairable and extended FTs. An adapted form of Shannon's decomposition theory merged with Dynamic Binary Decision Tree (DBDT) has been proposed by [50] to solve DFT. The proposed method increased the computational efficiency. However, this PAND gate and the method was not generalized for other dynamic gates. In the same year [51] proposed a models called "AltaRica" to reduce the state explosion through combining the Dijkstra's algorithm and notion of distance factor. An approximate solution for DFT through truncating Markov chain states has been presented in 2016 by [52] .

An automated tool for the valuation of repairable DFT has been presented by [53]. The paper proposed a mapping from DFT entity to adaptive transition system entity, and a conception of failure gates for the evaluation of both reliability and availability has been illustrated. This paper used the SMP for reliability evaluation. However, their methodology of solving SMP and DFT is different from the methodology of this paper. The existing differences and categories in DFT variants has been studied by [54]. The research emphasized that those reviewed differences may affect the reliability evaluation and analysis. A hierarchical and approximate solution for availability analysis in DFTs based on equivalent two-state Markov models has been proposed by [55]. Their approach was only tailored exponential failure distribution based events. Reference [56] focused on priority AND gate and analyzed the region of coherence in this gate. The outcome of the study was to determine the coherence bound of PAND gate and improve the efficiency of the dynamic dependability evaluation process.

The method was appropriate for both reparable and nonreparable systems. In 2017 the research work of [50] has been extended and published in [57]. The research has covered spare and sequence gates through De Morgan theorem, and for negating a generalized cut sequences, they have improved explicit formula. Reference [58] has provided a framework to do qualitative and quantitative analysis of the DFT through Generalized Boolean logic Driven Markov Processes (GBDMP). In 2018, a new state-space generation approach for solving the DFTs has been presented by [59]. The introduced approach has the capability of model reduction through model checking theories.

In spite of intensive research works conducted so far, there is no reported similar research work on the hierarchical semiMarkov based DFT solution. This paper presents a novel hierarchical SMP-based DFT solution for the first time with the following motivations. I) Providing a parametric solution that enables us to extract further parameters such as MTTF and Sensitivity. II) Considering the non-exponential failure distributions in a hierarchical manner. III) Reducing the computational complexity and especially state explosion in the models. IV) Considering repair events in DFT. Moreover, a universal SMP model for static and dynamic gates is introduced that can generalize dynamic behaviours like functional dependencies, sequences, priorities and spares in a single model.

\section{SMP ANALYTICAL SOLUTION}

In this section, analytical solution of SMP is addressed and then the reliability of PAND gate is calculated by this theorem. 


\section{A. Solution of SMP by the use of LST}

SMP can be modeled by different notations [60], This paper uses tuple (trio) ( $\mathrm{p}, \mathrm{P}, \mathrm{F}(\mathrm{t})$ ), where: $\mathrm{p}$ is vector of initial distribution, $\mathrm{P}$ is matrix of conditional transition probabilities and $F(t)$ describes matrix of distribution functions of sojourn times in state $i^{\text {th }}$, when $j^{\text {th }}$ state is next;

Considering $X_{i}, \forall i=0,1,2, \ldots$ as a random variable, the time-homogeneous SMP $\mathrm{X}$ is determined by a vector of initial state probabilities $p(0)=\left[P\left\{X_{0}=i\right\}\right]=[1,0, \ldots, 0]$, and the conditional transition probability matrix $P(t)=\left[P_{i j}(t)\right]$.

The conditional probabilities' matrix $\left(P_{i j}(t)\right)$ is satisfied by Kolmogorov-Feller's equations in (1) [61].

$$
P_{i j}(t)=\delta_{i j}\left[1-G_{i}(t)\right]+\sum_{K \in S} \int_{0}^{t} P_{k j}(t-x) d Q_{i k}(x)
$$

where $\delta_{i j}=1$ if $i=j$ and $\delta_{i j}=0$ otherwise, $G_{i}$ is the distribution of the sojourn time in state $i[62,63]$, and $Q_{i j}(t)$ describes the kernel matrix. Note that the kernel matrix has a Markov renewal theorem in the background [64].

Solution of (1) an be found by applying Laplace Stieltjes Transformation (LST) in (2) [65]. This is a set of Voltera equations in (1) which is a Markov renewal equation [62]. Note that for non-exponential failure distributions such as Weibull and Gamma, some approximation is needed (Refer to [66, 60, 2]).

$$
\tilde{p}_{i j}(s)=\delta_{i j}\left[1-\tilde{g}_{i}(s)\right]+\sum_{K \in S} \tilde{q}_{i k}(s) \tilde{p}_{k j}(s)
$$

Equation (2) in the matrix form can be rewritten as follows:

$$
\tilde{p}(s)=[I-\tilde{g}(s)]+\tilde{q}(s) \tilde{p}(s)
$$

Hence, it can be rewritten as (4) through simple algebraic replacement.

$$
\tilde{p}(s)=[1-\tilde{q}(s)]^{-1}(I-\tilde{g}(s))
$$

In (4), the inverse of $1-\tilde{q}(s)$ can be replaced by the summation of powers of $\tilde{q}(s)$. The resulted equation which is useful for a singular kernel matrix will be as (5).

$$
\tilde{p}(s)=\left(\sum_{n=0}^{\infty} \tilde{q}(s)^{n}\right)(I-\tilde{g}(s))
$$

Having solved (5) with taking the inverse LST of $\tilde{p}(s)$, the unconditional state probabilities in time domain are determined as follows:

$$
P(t)=P(0) P(t)
$$

Finally, the reliability of system can be achieved by summing of the transient probability of operational states.

\section{B. Reliability Evaluation of PAND Gate through SMP}

The Markov model of PAND gate has previously been presented in the literature. Fig 2 illustrates the semi-Markov model of PAND gate with any failure distribution function. It can be solved by SMP theorem. In this model, $F_{A}(t)$ is Cumulative Distribution Function (CDF) of the first input of PAND gate and $F_{B}(t)$ is CDF of the second input of PAND gate respectively. States are numbered from 1 to 5 from top to bottom and left to right. This model can be solved by SMP theorem described in the previous sub-section.

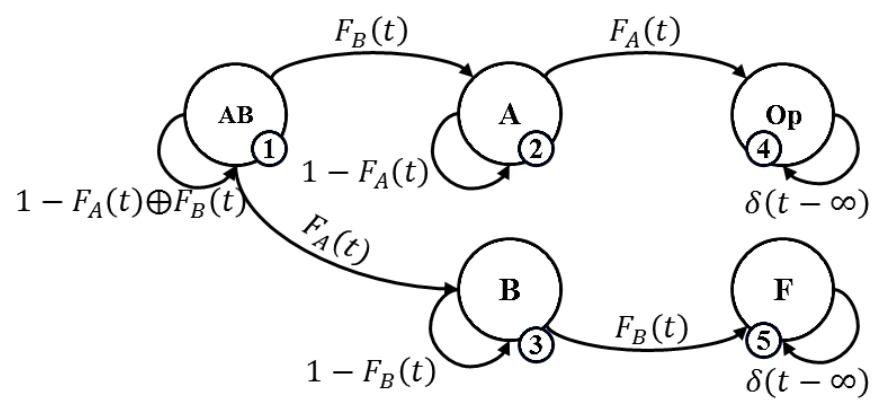

Fig. 1. Semi-Markov model of PNAD gate

The kernel matrix of Fig. 2 can be written in the form of (7) and distribution matrix of the sojourn time in each state is in the form of (8).

$$
Q(t)=\left[\begin{array}{ccccc}
0 & Q_{1,2} & Q_{1,3} & 0 & 0 \\
0 & 0 & 0 & Q_{2,4} & 0 \\
0 & 0 & 0 & 0 & Q_{3,5} \\
0 & 0 & 0 & 0 & 0 \\
0 & 0 & 0 & 0 & 0
\end{array}\right]
$$

The dimension of both kernel matrix and sojourn distribution matrix should be $\mathrm{n}$ by $\mathrm{n}$ where $\mathrm{n}$ is the number of states.

$$
\begin{array}{r}
G(t)=\operatorname{diag}\left(F_{A}(t) \oplus F_{B}(t), F_{A}(t),\right. \\
\left.F_{B}(t), \delta(t-\infty), \delta(t-\infty)\right)
\end{array}
$$

where the $\oplus$ operation is defined by (9). In other words this is a probabilistic OR algebraic operation.

$$
A \oplus B=1-(1-A)(1-B)
$$

In matrix (7), $Q_{1,2}$ is the system failure probability up to time $t$, if $B$ is occurred at first. It can be written as (10). Subscript 1,2 stands for "from state one to state two".

$$
\begin{aligned}
Q_{1,2} & =\operatorname{Pr}\left\{\mathrm{X}_{1}=2, S_{1} \leq t \mid X_{0}=1\right\} \\
& =\operatorname{Pr}\left\{\mathrm{L}_{B} \leq t \wedge L_{A} \leq L_{B}\right\}=\int_{0}^{t} 1-F_{A}(t) d F_{B}(t)
\end{aligned}
$$

For exponential failure behavior case of A and B (10) can be written as (11). where $\lambda_{A}$ and $\lambda_{B}$ are the constant failure rates of events or inputs $\mathrm{A}$ and $\mathrm{B}$ respectively.

$$
\begin{aligned}
Q_{1,2} & =\int_{0}^{t}\left[1-\left(1-e^{-\lambda_{A} \tau}\right)\right] d\left\{1-e^{-\lambda_{B} \tau}\right\} \\
& =\int_{0}^{t} e^{-\left(\lambda_{A}+\lambda_{B}\right) \tau} \lambda_{B} d \tau=\frac{\lambda_{B}\left[1-e^{-\left(\lambda_{A}+\lambda_{B}\right) t}\right]}{\lambda_{A}+\lambda_{B}}
\end{aligned}
$$

Similarly, $Q_{1,3}$ is the system failure probability up to time t, in a case that $\mathrm{A}$ is occurred sooner. It means the related occurrence time of event $\mathrm{A}$ is less than related occurrence time 
of event B. It can be written as (12). For exponential failure distribution function of both inputs, (12) can be written as (13).

$$
\begin{aligned}
& Q_{1,3}=\operatorname{Pr}\left\{\mathrm{X}_{1}=3, S_{1} \leq t \mid X_{0}=1\right\} \\
& =\operatorname{Pr}\left\{\mathrm{L}_{A} \leq t \wedge L_{B} \leq L_{A}\right\}
\end{aligned}
$$

Similarly, $\lambda_{A}$ and $\lambda_{B}$ are the constant failure rates of events or inputs $\mathrm{A}$ and $\mathrm{B}$ respectively.

$$
\begin{aligned}
Q_{1,3} & =\int_{0}^{t}\left[1-\left(1-e^{-\lambda_{B} \tau}\right)\right] d\left\{1-e^{-\lambda_{A} \tau}\right\} \\
& =\int_{0}^{t} e^{-\left(\lambda_{A}+\lambda_{B}\right) \tau} \lambda_{A} d \tau=\frac{\lambda_{A}\left[1-e^{-\left(\lambda_{A}+\lambda_{B}\right) t}\right]}{\lambda_{A}+\lambda_{B}}
\end{aligned}
$$

$Q_{2,4}$ is the failure probability of input or event A before or at time $\mathrm{t}$ and $Q_{3,5}$ is the failure probability of input or event B before or at time t. $Q_{2,4}$ and $Q_{3,5}$ are defined by (14) and (15), respectively.

$Q_{2,4}=\operatorname{Pr}\left\{\mathrm{X}_{1}=4, S_{1} \leq t \mid X_{0}=2\right\}$

$=\operatorname{Pr}\{A$ fails before or at time $\mathrm{t}\}$

$$
=\operatorname{Pr}\left\{\mathrm{L}_{A} \leq t\right\}
$$

Note that 2,4 denotes "from state two to state 4" and 3,5 denotes "from state three to state five".

$Q_{3,5}=\operatorname{Pr}\left\{\mathrm{X}_{1}=3, S_{1} \leq t \mid X_{0}=5\right\}$

$=\operatorname{Pr}\{\mathrm{B}$ fails before or at time $\mathrm{t}\}$

$=\operatorname{Pr}\left\{\mathrm{L}_{B} \leq t\right\}$

In exponential form (14) and (15) are written by (16) and (17), respectively.

$Q_{2,4}==\int_{0}^{t} \lambda_{A} e^{-\lambda_{A} \tau} d \tau=1-e^{-\lambda_{A} t}$

It should be noted that both failure rates are constant.

$$
Q_{3,5}==\int_{0}^{t} \lambda_{B} e^{-\lambda_{B} \tau} d \tau=1-e^{-\lambda_{B} t}
$$

The LST of kernel matrix is written as (18) in its general form. As can be seen, in this matrix, there are four nonzero transition.

$$
\tilde{q}(s)=\left[\begin{array}{ccccc}
0 & L\left\{Q_{1,2}\right\} & L\left\{Q_{1,3}\right\} & 0 & 0 \\
0 & 0 & 0 & f_{A}^{*}(s) & 0 \\
0 & 0 & 0 & 0 & f_{B}^{*}(s) \\
0 & 0 & 0 & 0 & 0 \\
0 & 0 & 0 & 0 & 0
\end{array}\right]
$$

In general form, the LST of G matrix is written as (19). In exponential form, it is written as (20). Note that "diag" creates diagonal matrix from each input vector.

$$
\tilde{g}(s)=\operatorname{diag}\left(L\left\{F_{A}(t) \oplus F_{B}(t)\right\}, f_{A}^{*}(s), f_{B}^{*}(s), 1,1\right)
$$

It is assumed that the failure rates are constant in the following equation.

$$
I-\tilde{g}(s)=\operatorname{diag}\left(\frac{s}{s+\lambda_{A}+\lambda_{B}}, \frac{s}{s+\lambda_{A}}, \frac{s}{s+\lambda_{B}}, 1,1\right)
$$

Similarly, (21) is LST of kernel matrix for exponential failures.

$$
\begin{aligned}
& \tilde{q}(s)= \\
& {\left[\begin{array}{ccccc}
0 & \frac{\lambda_{B}}{s+\lambda_{A}+\lambda_{B}} & \frac{\lambda_{A}}{s+\lambda_{A}+\lambda_{B}} & 0 & 0 \\
0 & 0 & 0 & \frac{\lambda_{A}}{s+\lambda_{A}} & 0 \\
0 & 0 & 0 & 0 & \frac{\lambda_{B}}{s+\lambda_{B}} \\
0 & 0 & 0 & 0 & 0 \\
0 & 0 & 0 & 0 & 0
\end{array}\right]}
\end{aligned}
$$

By the use of (4) or (5), the unconditional probability vector of Fig. 2 is computed as (22).

$$
\begin{aligned}
& \hat{P}=\left[L^{-1}\left\{\left(1-f_{A}^{*}(s)\right)\left(1-f_{B}^{*}(s)\right)\right\},\right. \\
& L^{-1}\left\{q_{1,2}(s)\left(1-f_{A}^{*}(s)\right)\right\},, L^{-1}\left\{q_{1,3}(s)\left(1-f_{B}^{*}(s)\right)\right\} \\
& \left.L^{-1}\left\{q_{1,2}(s)\left(f_{A}^{*}(s)\right)\right\}, \quad L^{-1}\left\{q_{1,3}(s)\left(f_{B}^{*}(s)\right)\right\}\right]
\end{aligned}
$$

Finally, reliability of PAND gate can be obtained from the probability of fail state (state 5 in Fig. 1) using (23). We named this equation as "general equation of PAND gate", Note that, this paper defines a general equation for each dynamic gate of a given DFT obtained by SMP. Having implemented the general equation of all gates, a library is constructed (Fig. 3). This library can be used to create the reliability equation of any given gate with any CDF. For example, the reliability of a PAND gate with exponential CDF can be extracted from this library. If Gate Type is selected "PAND" and CDF Type is chosen "Exponential", the outcome will be as (27). This library can be used by DFT users to find the reliability of given DFT as explained in section IV. This library can be used as a library for DFT users for reliability evaluation proposes [67].

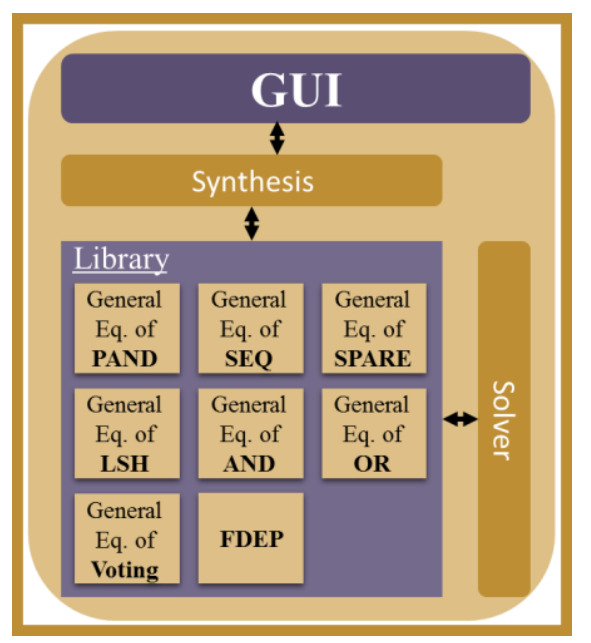

Fig. 2. Procedure that happened inside of library of each dynamic gate 


$$
R(t)=1-F(t)=1-L^{-1}\left\{q_{1,3}(s)\left(f_{B}^{*}(s)\right)\right\}
$$

The final reliability expression shows the consistency of the results with the existing one in the literature.

$$
\begin{aligned}
& R(t)=1-F(t)=1-L^{-1}\left\{\left(\frac{\lambda_{A}}{s+\lambda_{A}+\lambda_{B}}\right)\left(\frac{\lambda_{B}}{s+\lambda_{B}}\right)\right\} \\
& \Rightarrow R(t)=e^{-\lambda_{B} t}+\frac{\lambda_{B}\left(1-e^{-\left(\lambda_{A}+\lambda_{B}\right) t}\right)}{\lambda_{A}+\lambda_{B}}
\end{aligned}
$$

\section{The Novel Universal Semi-Markov Model of DFT gates}

Fig. 3 shows the universal representation of a dynamic or static gate with inputs A and S. Its SMP model with any CDF will be as Fig. 4.

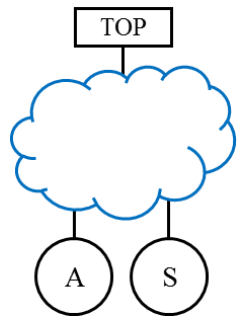

Fig. 3. Typical static or dynamic gate

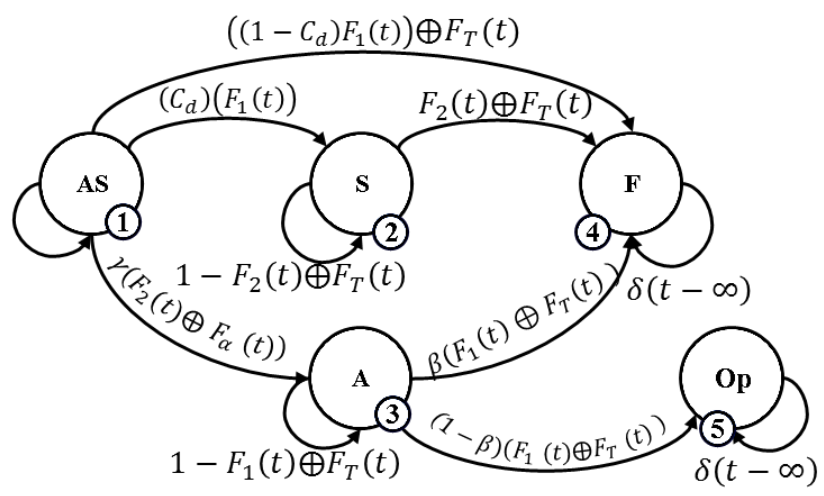

$\mathrm{A} \oplus B=1-(1-A)(1-B)$

Fig. 4. Universal semi-Markov model of static and dynamic gates gate

The universal model of Fig. 4 is a five states SMP with states AS, A, S, F and Op. The state AS denotes that both input of the gate are working correctly. The state S or A denotes that only one of the inputs of the gate works correctly. The state $\mathrm{F}$ shows the failing of the system and the state Op shows correct functioning of the system in spite of the failure of its both inputs (This case occurs where one of the inputs has a supportive or protective mechanism to the other). Note that we have assumed that $\mathrm{A}$ is the first input of the gate and $\mathrm{S}$ is the second. It is obvious that the use of this universal model (Fig. 4) speeds-up the modelling and solution of dynamic gates.

This paper claims that the introduced universal model has the potential to describe some of the conventional static and dynamic gates by means of four probabilistic parameters $\alpha, \beta, \gamma, C_{d}$, and state transitions CDFs. The parameter $\alpha$, called "dormancy factor" in this paper, eliminates the lower states of the model (states A and Op). The parameter $\beta$, called
"Non-PAND factor" in this paper determines whether the investigated gate is PAND-type; where $\beta=0$ the gate is PAND-type, where $\beta=1$ gate is semi-PAND-type [67]. The parameter $\gamma$, called "Non-Sequence factor", in this paper determines whether investigated gate is sequence-type gate; when $\gamma=0$ the gate is sequential-type and when $\gamma=1$ the gate can be sequential-type. The parameter $C_{d}$ indicates the probability of switching mechanism of the SPARE type gates (CSP, WSP and HSP).

In Fig. $4, F_{1}(t)$ is the failure $\mathrm{CDF}$ of input $\mathrm{A}, F_{2}(t)$ is the failure $\mathrm{CDF}$ of input $\mathrm{B}, F_{T}(t)$ is the $\mathrm{CDF}$ of a triggered failure which affects failing of both $\mathrm{A}$ and $\mathrm{S}$ (will be explained in this paper later). In fact, the triggering event of this type is taken as a fatal shock [68]. $F_{\alpha}(t)$ has been used in the model for indicating the CDF of the second input's dormancy which is a time-dependent variable (named $\alpha$ in this paper) has a direct impact on $F_{\alpha}(t)$. For this reason $\alpha$ has not been directly used in the model of Fig. 4.

\section{Explanation of the Novel Model Structure}

The model starts from state AS in which gate inputs A and S are working correctly. By i) failing of A with failure distribution $F_{1}(t)$ the system transits to state $\mathrm{S}$ if the switching mechanism performs perfectly or ii) goes to state $\mathrm{F}$ if either the switching mechanism acts imperfectly or iii) the trigger event (if exists) affects A with the CDF $F_{T}(t)$.

Similarly, the system goes from state $\mathrm{S}$ to state $\mathrm{F}$ with failure distribution $F_{2}(t) \oplus F_{T}(t)$. The system goes from state AS to $A$ if the second input $S$ is either i) underworking condition and subjected to fail with failure distribution $F_{2}(t)$ or ii) partly underworking with $F_{\alpha}(t)$. The performance of sequence gate can clarify this issue. The parameter $\gamma$ represents the existence of occurrence sequence for gate inputs such that $\gamma=0$ : sequence exists and $\gamma=1$ : no sequence exists. The parameter $\beta$ represents the existence of priority for gate inputs such that $\beta=1$ : no priority exists and $\beta=0$ : priority exists.

\section{E. Examples of Universal Usage}

In this subsection, we show how to extract the model of a given gate from the universal gate model.

\section{1) Static OR gate}

This gate has:

- No switching mechanism, thus $C_{d}=1$.

- No sequence for the failure of its inputs, thus $\gamma=1$.

- No dormancy for its second input thus, $\alpha=0$ and $F_{\alpha}(t)$ exists

- No priority for the failure of its inputs, thus $\beta=1$.

- No dependency on external trigger for the failure of its inputs, thus $F_{T}(t)=0$.

Therefore, the universal model of Fig. 4 is simplified as Fig. 5 
for OR gate.
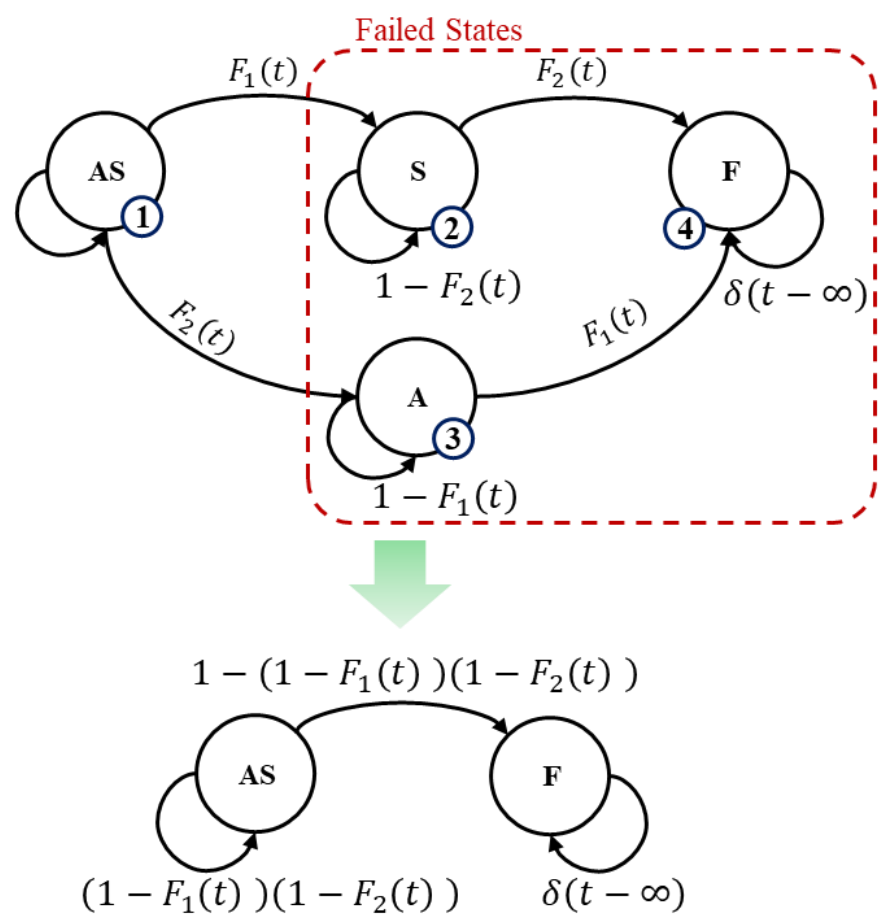

$$
\left(1-F_{1}(t)\right)\left(1-F_{2}(t)\right) \quad \delta(t-\infty)
$$

Fig. 5. Obtained Static OR gate from universal Semi-Markov model and its simplification

It is obvious that the reliability of this gate is $P_{O R}(t)=P_{A S}(t)$, where $P_{A S}(t)$ is the probability of state AS at the time interval $\mathrm{t}$.

\section{2) Dynamic $S E Q$ gate}

This dynamic gate has:

- No switching mechanism thus $C_{d}=1$.

- No sequence for the failure of its inputs thus $\gamma=0$.

- No dormancy for its second input thus $\alpha=0$ and $F_{\alpha}(t)=0$.

- No priority for the failure of its inputs thus $\beta=1$.

- Dependency on external trigger for the failure of its inputs thus $F_{T}(t)$ exists.

Therefore, the universal model of Fig. 4 is simplified as Fig. 6 for SEQ gate.

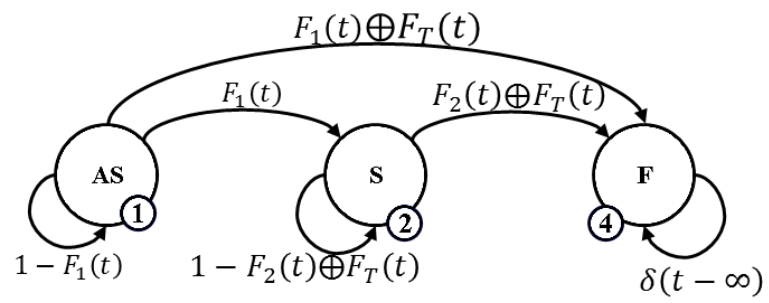

Fig. 6. Semi-Markov model of dynamic SEQ gate with triggered inputs achieved from the universal semi-Markov model

\section{F. Discussion on Universal Model}

In the literature, in spite of considerable attempts there is no universal semi-Markov model for dynamic and static gates.
This paper introduced, for the first time, a universal semiMarkov model for static and dynamic gates in which by assigning model parameters the model of any given gate can be obtained. TABLE I indicates this issue. It shows that by selecting $\alpha=0, \beta=1, \gamma=1$ and $C_{d}=1$ the universal gate model is converted to OR gate model. Other possibilities for selecting model parameters to extract the model of different known gates are shown in this table. In the introduced model (Fig. 4) $F_{1}(t)=F_{4}(t)$ and $F_{2}(t)=F_{3}(t)$ except for Load Sharing gate (LSH).

In this table, it is assumed that the switching mechanism is perfect except SPARE gates (CSP, WSP and HSP). In addition, $\beta$ factor is used to describe PAND gate. For PAND gate both $\alpha$ and $\beta$ are zero. The LSH gate has been defined to reshape and solve DFT with shared SPARE gates. In LSH gate transition $\operatorname{CDFs} F_{1}(t), F_{2}(t), F_{3}(t)$ and $F_{4}(t)$ can be chosen independently. i.e. $F_{1}(t)$ may take Weibull form while $F_{2}(t)$ can be exponential form (see appendix). Reliability of OR gate is obtained by probability of state AS (denoted here $P_{S 1}$ ) and the reliability of other gates is achieved by $1-P_{S 4}$ in which $P_{S 4}$ is the probability of state $\mathrm{F}$ of the model. In TABLE I, "X" is used to represents cases in which a parameter has no impact on the model.

Note that this table does not consider FDEP gate because we have inserted directly the "functional dependency" property of FDEP gate into the universal model. In other words, we claim that it is not necessary to build a separated Markov model for FDEP gate, instead we have considered the functional property of this gate directly into the model. See Fig. 7, how we considered $F_{T}(t) \mathrm{CDF}$ in the state transitions of universal model.

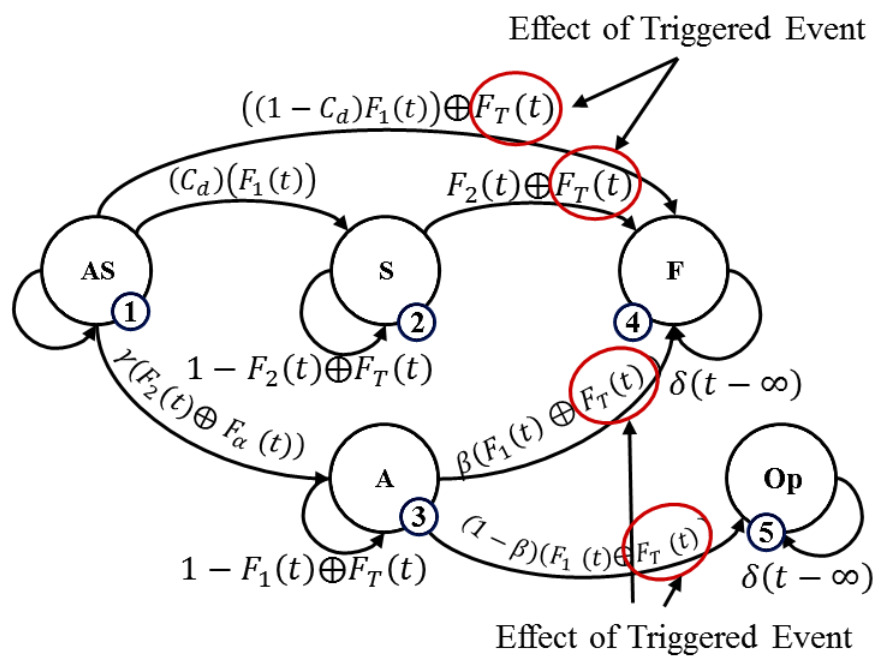

Fig. 7. Consideration of trigger effect of event in universal model

This idea is depicted in Fig. 8. Fig. 8-a. is replaced by Fig. 8b. The symbol A $\mid \mathrm{T}$ denotes the event A. $(\mathrm{A}+\mathrm{T})=\mathrm{A}+\mathrm{AT}$. 


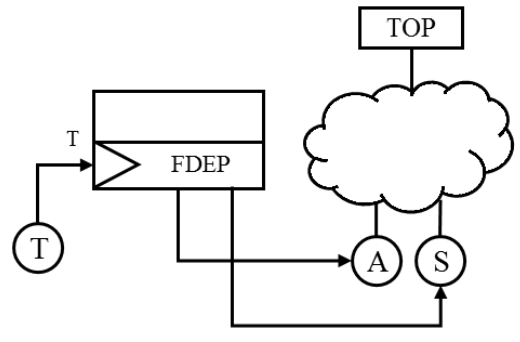

(a)

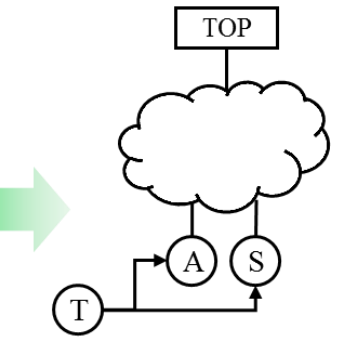

(b)

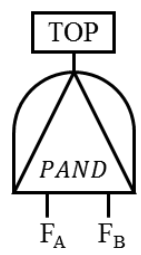

(a)

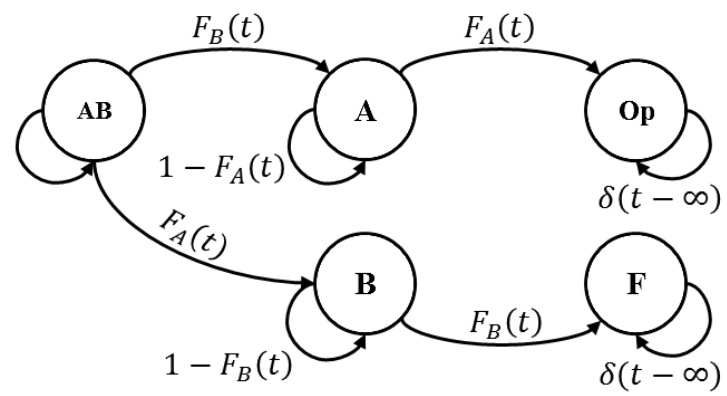

Fig. 8. The idea of FDEP gate omission in DFT

To clarify this issue, we show how in Fig. 9 Markov model of PAND gate with ordinary inputs and Markov model of a PAND gate with triggered inputs (via a FDEP gate) both extracted from universal model. The idea of universal gate is also extendable for Probabilistic Dependency (PDEP) gate.

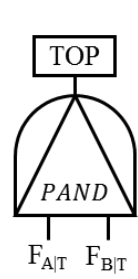

(b)

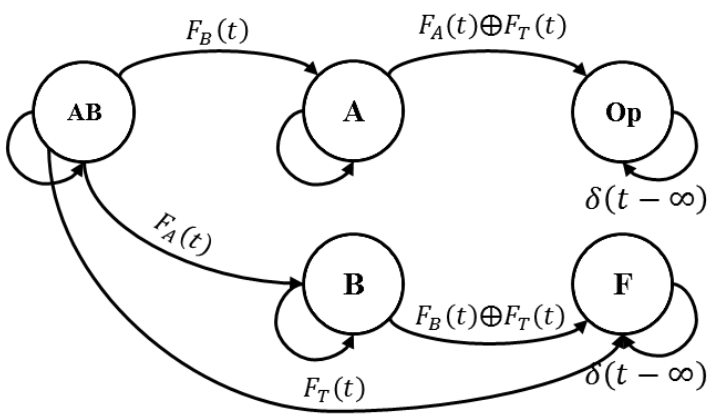

Fig. 9. (a) PAND gate without triggered event, (b) PAND gate with triggered event.

TABLE I

PARAMETER OF UNIVERSAL GATE

\begin{tabular}{|c|c|c|c|c|c|c|}
\hline Gates & $\begin{array}{c}\text { Dormancy } \\
\text { Factor }(\alpha)\end{array}$ & $\begin{array}{c}\text { Priority } \\
\text { Factor }(\beta)\end{array}$ & $\begin{array}{c}\text { Sequence } \\
\text { Factor }(\gamma)\end{array}$ & $\begin{array}{c}\text { Switching } \\
\text { Factor }\left(C_{d}\right)\end{array}$ & CDF & Reliability \\
\hline OR & 0 & 1 & 1 & 1 & $F_{1}(t)=F_{4}(t), F_{2}(t)=F_{3}(t)$ & $R(t)=P_{S 1}$ \\
\hline PAND & 0 & 0 & 1 & 1 & $F_{1}(t)=F_{4}(t), F_{2}(t)=F_{3}(t)$ & $R(t)=1-P_{S 4}$ \\
\hline POR & 0 & 0 & 1 & 1 & $F_{1}(t), F_{2}(t), F_{3}(t)=F_{4}(t)=1$ & $R(t)=1-P_{S 4}$ \\
\hline WSP & $\alpha$ & 1 & 1 & {$\left[\begin{array}{ll}0 & 1\end{array}\right]$} & $F_{1}(t)=F_{4}(t), F_{2}(t)=F_{3}(t)$ & $R(t)=1-P_{S 4}$ \\
\hline HSP & 0 & 1 & 1 & {$\left[\begin{array}{ll}0 & 1\end{array}\right]$} & $F_{1}(t)=F_{4}(t), F_{2}(t)=F_{3}(t)$ & $R(t)=1-P_{S 4}$ \\
\hline SEQ & $\mathrm{X}$ & $\mathrm{X}$ & 0 & 1 & $F_{1}(t), F_{3}(t), F_{2}(t)=F_{4}(t)=X$ & $R(t)=1-P_{S 4}$ \\
\hline Load Sharing & 0 & 1 & 1 & 1 & $F_{i}(t), i=1, \ldots, 4$ are independent & $R(t)=1-P_{S 4}$ \\
\hline
\end{tabular}

\section{G. General Reliability Equation of Dynamic Gates}

It is now obvious that by the use of TABLE I and universal Markov model (Fig. 4), we can build the Markov model of any gate. For example, for SEQ gate, according to TABLE I: the Markov model will be as Fig. 10 extracted from Fig. 4.

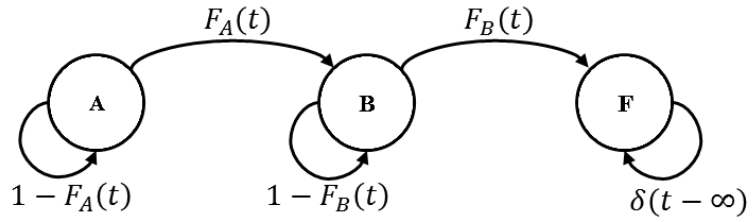

Fig. 10. Semi-Markov model of SEQ gate with two inputs

The general reliability equation of this model, by the use of SMP theorem explained in section III is obtained as follow:

$$
\begin{aligned}
& R(t)=1-L^{-1}\left\{q_{1,2}(s) q_{2,3}(s)\right\} \\
& =1-L^{-1}\left\{\left(f_{A}^{*}(s)\right)\left(f_{B}^{*}(s)\right)\right\}
\end{aligned}
$$

The second term of this equation is indeed the CDF of gate output. Similarly, general equation of SPARE gates for reliability evaluation is achieved by

$$
\begin{aligned}
& R(t)=1- \\
& L^{-1}\left\{q_{1,4}(s)+q_{1,3}(s)\left(f_{A}^{*}(s)\right)+q_{1,2}(s)\left(f_{S}^{*}(s)\right)\right\}
\end{aligned}
$$

The general reliability equation of other gates can be easily obtained in a similar way, explained above.

\section{A HIERARCHICAL SMP-BASED DFT SOLUTION}

Dynamic Fault Trees (DFTs) was introduced, for the first time, by Dugan and Boyd (Ref. [21]) in which the whole DFT converted into a Markov model, and consequently, model 
explosion is resulted in. The problem was that the solution of this model was difficult and time-consuming. Later, a number of techniques based on Bayesian Networks, Petri Nets, Algebraic and etc. are presented for solving DFTs. In this article we present a hierarchical solving method for DFTs, based on SMP theorem.

\section{A. The Basic Idea}

First, the given DFT divided into a number of layers $(n)$. The lowest layer includes basic events and their associated gates. The outputs of the lowest layer form the inputs of first layer.
The first layer contains a number of gates, the outputs of these gates form the input of second layer ... the outputs of $(n-1) t h$ layer give the inputs of ( $n$ )th layer (Top layer). Starting from first layer, the gates of each layer are solved based on the method explained in previous sections to find their output CDF. The CDFs are used as $F(t)$ of inputs of the next layer. This procedure continuous until the CDF of Top event is obtained. It is now obvious that $R(t)=1-$ output $C D F$. The procedure is depicted in Fig. 11.
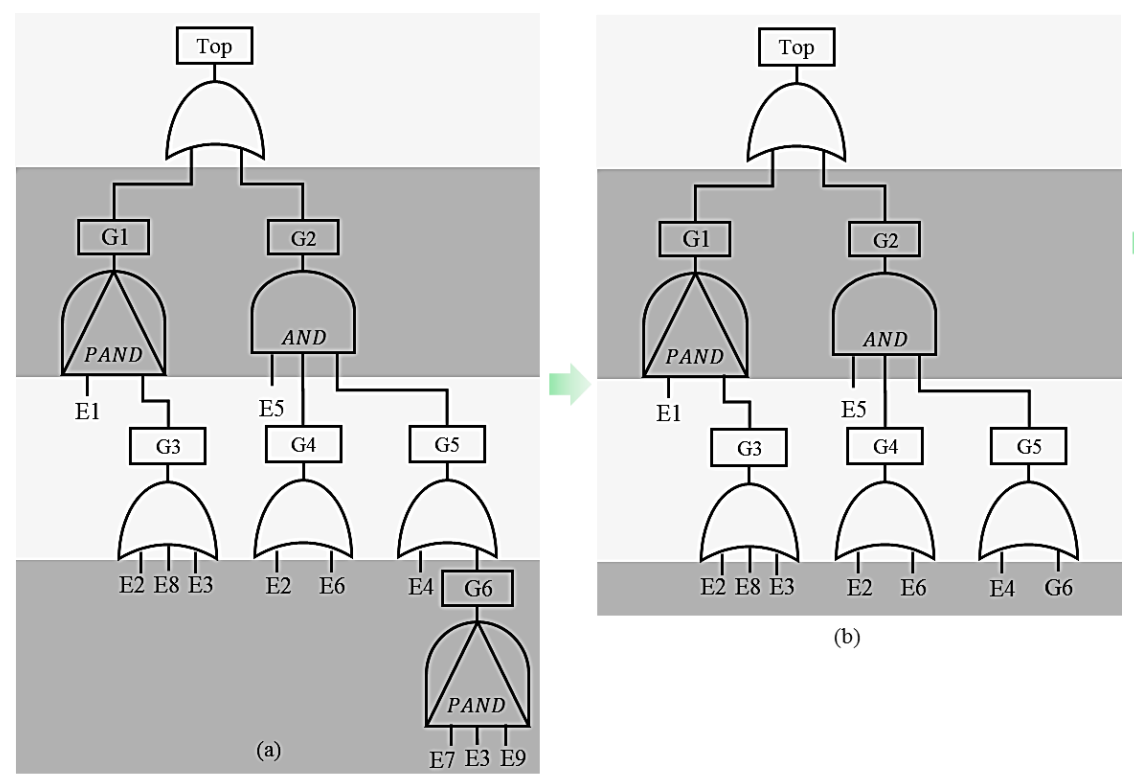

(b)
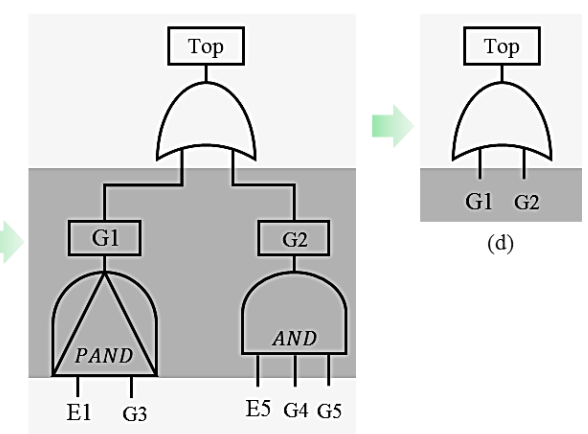

(d)

Fig. 11. An example of solving DFT through proposed method

The following issue most be considered in the implementation of this procedure for decreasing the volume of computations.

- Dynamic gates are separated from static gate. This is because solving static gates does not need SMP theorem. They can be easily solved by the use of conventional probability theorem.
- The solution of first layer gates can be easily carried out if their inputs have exponential CDF because gates with inputs with exponential CDF are solved with Markov theorem rather than SMP theorem. This consideration decreases the volume of computations.

Based on these issues the flow chart of SMP-based DFT's reliability solution will be as Fig. 12. 


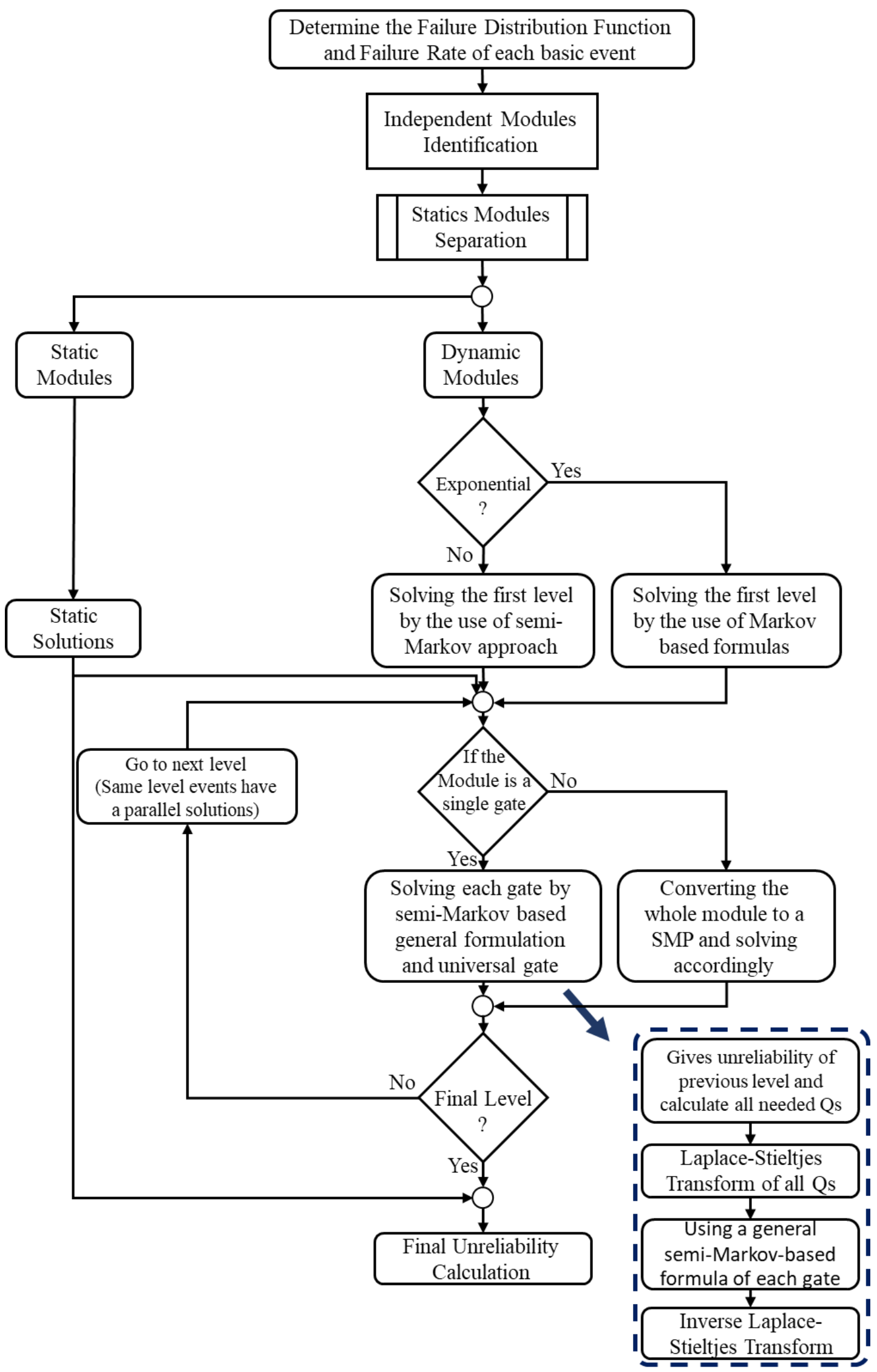

Fig. 12. Flow chart of SMP-based DFT's reliability solution (the proposed method of this paper) 


\section{EXAMPLES}

In this section, six examples are given to indicate the capabilities of the proposed method. The first example makes a comparison between the results of the proposed method and those obtained from the Windchill Quality Solution (WQS) software. The challenge point in this example is precision of the result in comparison with commercial tools. The second example studies the ability of this method applied in a DFT with repeated events. So, the challenge point of this example is about dealing with repeated events. In the third example, we will apply the proposed method on HCAS and compare the maximum number of states and transitions in three selected Markov-based DFT's solution approach. The robustness to state and transition explosion will be the challenge point of this example. The fourth example solves a selected DFT with event with exponential and non-exponential CDF, and then compares the results taken from the proposed method, and algebraic technique. Dealing with non-exponential CDF can be a challenge in reliability evaluation that is considered in example four. As the repair consideration can be a challenging issue in DFT, the fifth example discusses on the possibility of using proposed method for repairable DFTs. The final example deals with a case study of Aircraft Fuel Distribution System (AFDS).

\section{A. Example 1. DFT with a PAND Gate and Static Gates}

From the DFT in Fig14. it can be seen that this DFT consists of a PAND gate, two static gates and 10 basic events. Solving this DTF by the use of Markov theorem requires solving a CTMC with 160 states $[3,34]$. In contrast, the proposed method can solve this DFT through SMP theorem with only 5 states, it can even provide the parametric expression for reliability. Metrics such as sensitivity and MTBF can easily be evaluated through parametric results of the proposed method.

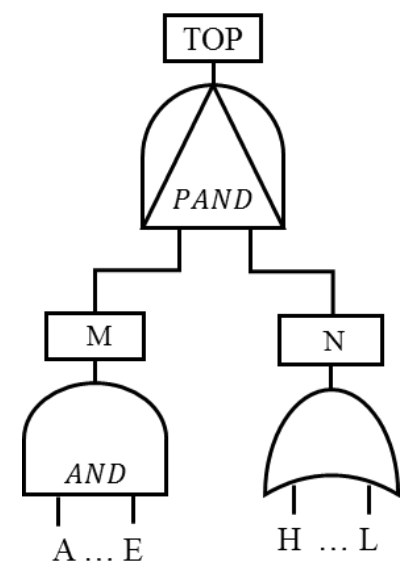

Fig. 13. DFT of the example 1. A tree with PAND gate, two static gates and 10 basic events [3].

The failure rates of the above DFT's events have been listed in Table II. In this table, failure rates are "failure per hour".

TABLE II

UNITS FOR MAGNETIC PROPERTIES [34]

\begin{tabular}{cccc}
\hline \hline Basic Events & Failure Rates & Basic Events & Failure Rates \\
\hline$\lambda_{A}$ & 0.0110 & $\lambda_{H}$ & 0.0011
\end{tabular}

\begin{tabular}{llll}
$\lambda_{B}$ & 0.0120 & $\lambda_{I}$ & 0.0012 \\
$\lambda_{C}$ & 0.0130 & $\lambda_{J}$ & 0.0013 \\
$\lambda_{D}$ & 0.0140 & $\lambda_{K}$ & 0.0014 \\
$\lambda_{E}$ & 0.0150 & $\lambda_{L}$ & 0.0015 \\
\hline
\end{tabular}

According to the result obtained in reference [34], the amount of unreliability of the system in 1000 hours of the mission time is 0.363 . The value obtained from our proposed method $(0.363024069761471)$ is completely coinciding with this result.

\section{B. Example 2. DFT with Repeated Basic Event}

This example examines the capability of the proposed method to model a DFT with repeated events. To do this the DFT of references [29, 3] which is shown in Fig. 14 is selected. This DFT consist of 9 basic events in which the event E2 is repeated. The failure rate of all basic events is set 0.01 failure per hour.

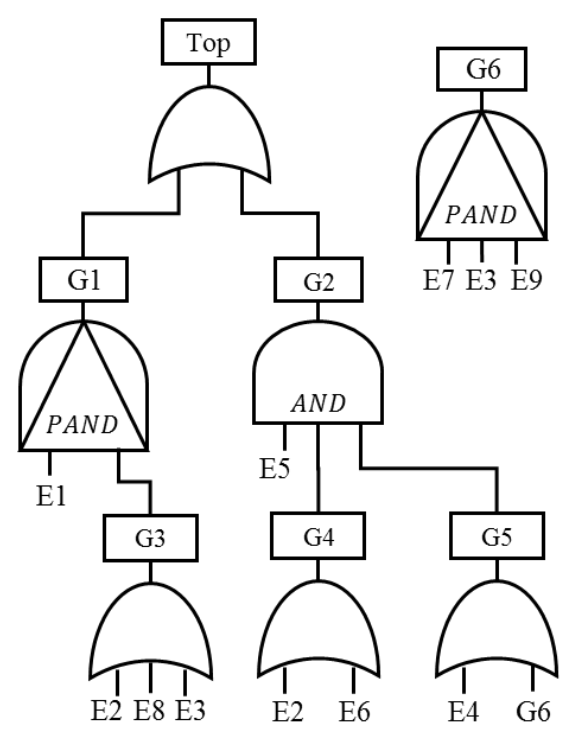

Fig. 14. DFT of example 2. A DFT with the repeated event [29]

Solving the above DFT in the time interval 0-300 hours has been provided through three methods in reference [29], using Galileo software, Monte Carlo simulation and a Yuge's method. Of these methods Galileo a more exact solution because its underlying approximation in the solution process of DFT is less than the others. This is why research works like [29] take the outcomes of Galileo as a reference. We solve the mentioned DFT through our proposed method, and the results are given along the results from the considered reference. The accuracy and precision of our method are then analyzed. Table III indicates the results. As seen the results of our method is closer to those of Galileo's results than other two methods (Yuge's method and Monte Carlo simulation).

TABLE III

COMPARING THE RESULTS OBTAINED FROM SOLVING DFT IN FIG. 14 OF REFERENCE [29] AND THE PROPOSED METHOD OF THIS PAPER

\begin{tabular}{ccccc}
\hline \hline Time & Galileo & Yuge's Method & Monte Carlo & Proposed method \\
\hline 0 & 0.00000 & 0.00000 & 0.00000 & 0.00000 \\
50 & 0.21418 & 0.25653 & 0.25647 & 0.19526
\end{tabular}




\begin{tabular}{lllll}
100 & 0.49318 & 0.59960 & 0.59970 & 0.45148 \\
150 & 0.68751 & 0.80196 & 0.80212 & 0.64738 \\
200 & 0.81010 & 0.90114 & 0.90120 & 0.78226 \\
250 & 0.88519 & 0.94864 & 0.94869 & 0.86873 \\
300 & 0.93066 & 0.97213 & 0.97215 & 0.92168 \\
\hline \hline
\end{tabular}

Fig. 15 shows the results graphically.

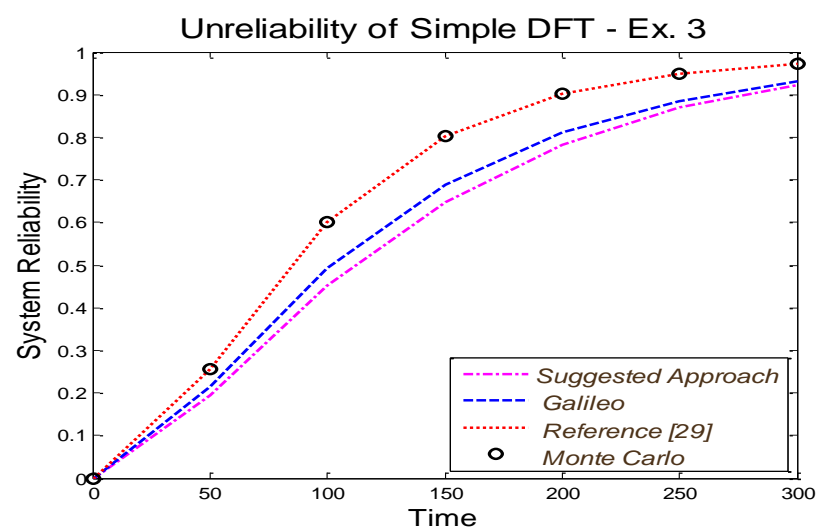

Fig. 15. The unreliability of the DFT in example 3 and comparing the results of the proposed method of this paper with results from reference [29].

Table IV indicates the results in terms of norm function. In this table, $Q_{s}$ stands for the unreliability results obtained from Monte Carlo simulation. $\mathrm{Q}_{\mathrm{G}}$ is the unreliability results obtained from Galileo and $\mathrm{Q}_{\mathrm{P}}$ denotes the unreliability results obtained from our proposed method. $\left\|Q_{P}-Q_{G}\right\|$ shows the difference between the unreliability of our proposed method and Galileo software.

TABLE IV

COMPARING DIFFERENCE NORM OF THE RESULTS IN RELIABILITY SOLUTION OF

\begin{tabular}{cccc}
\multicolumn{4}{c}{ DFT (EXAMPLE 3) } \\
\hline \hline$\left\|Q_{S}-Q_{G}\right\|$ & 0.45951 & 0.20080 & 0.11461 \\
$\left\|Q_{R}-Q_{G}\right\|$ & 0.45918 & 0.20062 & 0.11445 \\
$\left\|Q_{p}-Q_{G}\right\|$ & 0.15401 & 0.06951 & 0.04169 \\
\hline \hline
\end{tabular}

Table IV shows the superiority of our method to the others.

\section{Example 3. DFT of HCAS}

Hypothetical Cardiac Assist System (HCAS) is a benchmark in validating different methods applied to DFT reliability solution which has also been discussed in various references. The DFT of HCAS as shown in Fig. 16. including CPU module, motors module, and pumps module. This DFT consists of a shared CSP in the pump section. In another word to obtain a more exact output for this module of the DFT by means of our proposed method, this module must be reshaped. The new DFT after reshaping the pump module will be as Fig. 18 (see Appendix A. for related explanation). The new DFT uses an LSH gate which has been defined in section III of this paper.

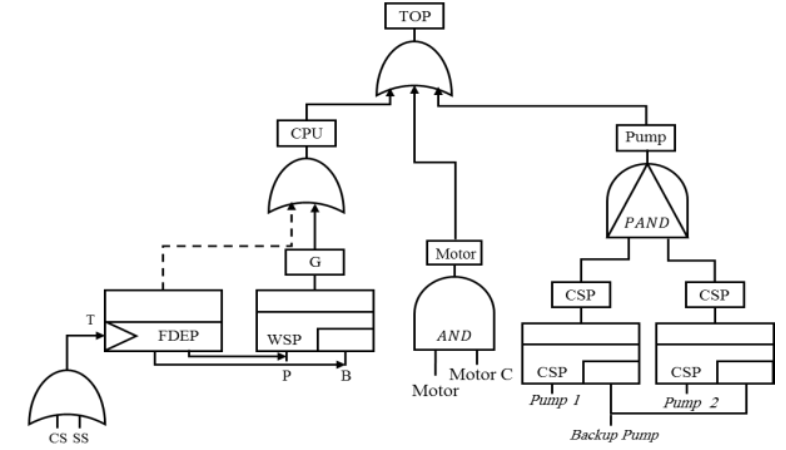

Fig. 16. DFT of HCAS with shared CSP gates in the pump module

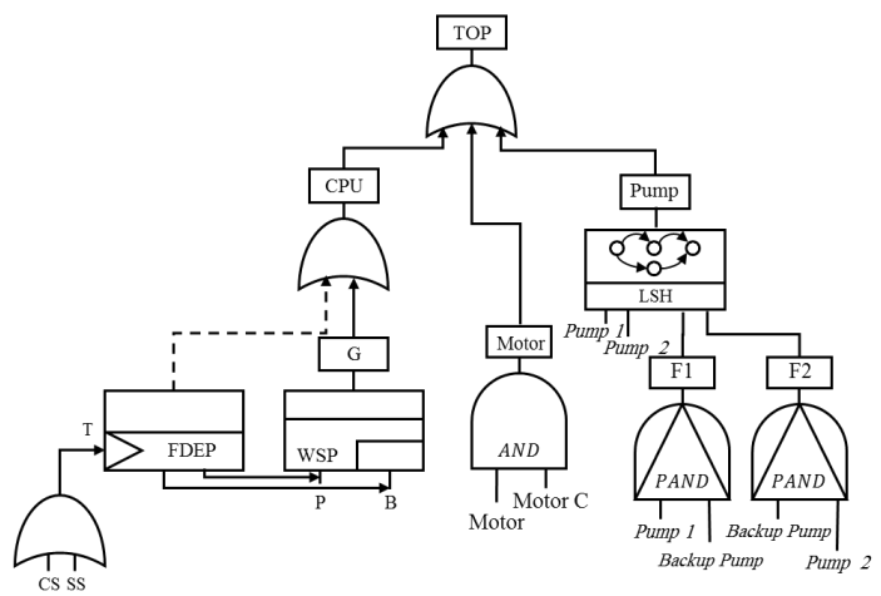

Fig. 17. DFT of HCAS with LSH gate in the pump module

The failure rates of basic events for this DFT have been assigned in Table V. Reference [69] has solved this DFT based on Bayesian Networks for 100000 mission time, and the system unreliability obtained 0.36501 .

TABLE V

FAILURE RATES OF BASIC EVENTS IN DFT OF HCAS IN EXAMPLE 4 [69]

\begin{tabular}{cccc}
\hline \hline $\begin{array}{c}\text { Basic } \\
\text { Events }\end{array}$ & $\begin{array}{c}\text { Failure Rates } \\
\left(10^{-6} \mathrm{~F} / \mathrm{hr}\right)\end{array}$ & Basic Events & $\begin{array}{c}\text { Failure Rates } \\
\left(10^{-6} \mathrm{~F} / \mathrm{hr}\right)\end{array}$ \\
\hline$C S$ & 1 & Pump $_{1}$ & 5 \\
$S S$ & 2 & Pump $_{2}$ & 5 \\
$P$ & 4 & Pump $_{\text {Backup }}$ & 5 \\
$B$ & 4 & Motor & 5 \\
MotorC & 1 & & \\
\hline \hline
\end{tabular}

The unreliability of this system computed from our proposed method is 0.363500847376541 which is more precise and in agreement with other research works' results. Another significant point in solving this model using the proposed method is decreasing the size of the corresponding Markov model of this DFT to clarify this consider Table VI. This table presents the number of states and the number of transitions of the corresponding Markov model of the largest module in DFT when solving by DIFtree, Coral and proposed method. As seen from the table, our proposed method possesses the lowest number of states and transitions. Generally speaking, the proposed method enables to decrease the number of states by the use of a reshaped model with load sharing gate. 
TABLE VI

A COMPARISON BETWEEN DIFFERENT MARKOV-BASED METHODS FOR DFT SOLUTION OF HCAS $[69,15]$

\begin{tabular}{lcc}
\hline \hline Analysis Method & Max. States Number & Max. Transitions Number \\
\hline DIFtree or Galileo & 8 & 10 \\
Coral & 36 & 119 \\
Proposed method & 5 & 5 \\
\hline \hline
\end{tabular}

As shown in Fig. 17, the DFT has eight gates and based on the proposed algorithm in each iteration only one gate will be solved through SMP and the CDF of the result will be stored to be used as an input for the next level gates. It means in each iteration the maximum number of states will never be higher than five based on the provided universal gate. Similarly, the maximum number of state transitions will never be higher than five as well. In other words, in HCAS DFT, there are two PANDs, one LSH, one AND, one WSP, one FDEP and three OR which means the maximum number of states will be used for evaluation of PAND gate (as shown in Fig. 9-a). In addition, the maximum number of state transition will be used for evaluation WSP with FDEP on its inputs (similar to Fig. 7).

The computational complexity of the Semi-Markov Reward Processes has been studied by [70] and if we consider the reward zero for operational states and reward one for failed state(s), the computational complexity of SMPs can be described as $\mathrm{O}\left(\left(\mathrm{x}^{[00]}+\mathrm{x}^{[01]}\right) \mathrm{n}\right)$ where $\mathrm{n}$ is the number of states, $\mathrm{x}^{[00]}$ is the number of nonzero transitions between operational states and $\mathrm{x}^{[01]}$ is the number of nonzero transitions from operational states to failed state(s). The proposed approach has a loop that evaluates the SMPs for each gate in the DFT. Thus, the computational complexity of the proposed method will be $\mathrm{O}\left(\left(\mathrm{x}^{[00]}+\mathrm{x}^{[01]} \mathrm{kn}\right)\right.$ where $\mathrm{k}$ is the number of gates in the fault tree. Based on the proposed universal gate, the maximum value of $\mathrm{x}^{[00]}+\mathrm{x}^{[01]}$ can be five and the maximum number of state can be also 5 . Therefore, because of bounded value in both number of states and number of transitions, the computational complexity of the proposed method is only depends on the number of iterations and can be simplified in $\mathrm{O}(\mathrm{k})$. On the other hand, the computational complexity of CTMC can be described as $\mathrm{O}\left(\mathrm{n}^{2}\right)$ [70] where $\mathrm{n}$ is the total number states. It is clear that the proposed approach has less computational complexity as it was expected than a traditional CTMC based approach.

D. Example 4. DFT with PAND gate and non-exponential failure distribution in its events

This example contains events with non-exponential CDF (Weibull CDF) to show the capability of the proposed method. The CDF of Weibull can be described by (27).

$$
F(t)=1-e^{-(t / \omega)^{\sigma}}
$$

where $\sigma$ is the shape and $\omega$ denotes the scale parameter of the Weibull CDF. First, consider the DFT of Fig. 13. The failure rate of basic events in this tree obeys exponential CDF with the values of Table II values except for J, K, and $\mathrm{L}$. It is assumed that the failure distribution of $\mathrm{J}, \mathrm{K}$, and $\mathrm{L}$ obeys Weibull CDF and the value of $\sigma$ is 0.1 and $\omega$ is 20. Fig. 18 shows the reliability of this DFT. As seen from this figure, both methods (proposed method and Algebraic method) gives exactly the same results.

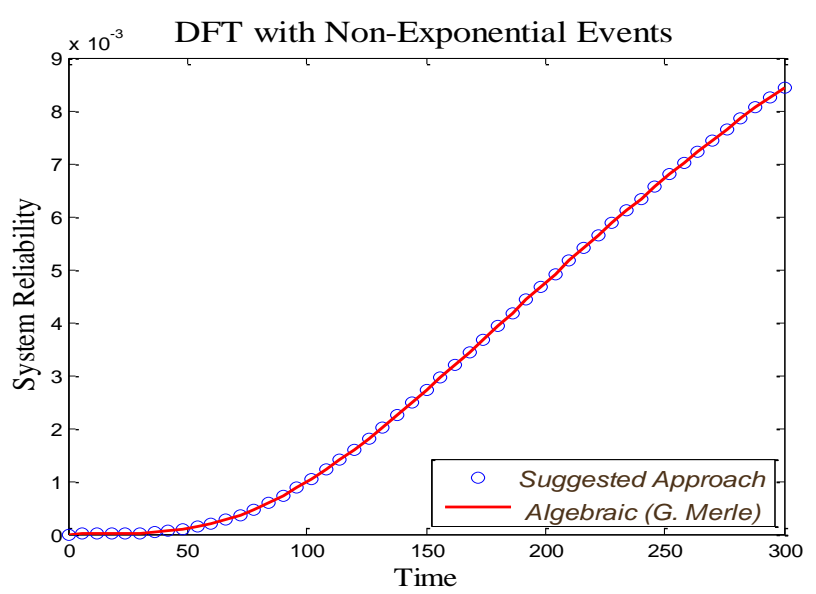

Fig. 18. Unreliability evaluation of the DFT of Example 4.

\section{E. Example 5. Repairable DFT}

The first generation of DFT was unable to repair modeling. References [71, 14, 72] presented an extended DFT able to model repair actions and solved it by the use of Generalized Stochastic Petri Net (GSPN) and Dynamic Bayesian Networks (DBN). This paper addresses the modelling of repair gate by a hypothetical example. It should be noted that in the current state we cannot guarantee that the proposed approach will work for any other repairable DFTs. In fact, this example is only a sign that there might be an opportunity to improve the proposed approach for repairable DFTs. The complete development of this modelling remains as future research work.

Through an example, we show that this modeling method is a good solution for finding the reliability of repairable DFT. Fig. 19 illustrates a repairable DFT with failure and repair rates of table VII.

TABLE VII

FAILURE RATES AND REPAIR RATES OF BASIC EVENTS IN REPAIRABLE DFT OF EXAMPLE 6 - EVENT C IS A NON-REPAIRABLE EVENT

\begin{tabular}{cccc}
\hline \hline $\begin{array}{c}\text { Basic } \\
\text { Events }\end{array}$ & $\begin{array}{c}\text { Failure Rates } \\
(\mathrm{F} / \mathrm{hr} .)\end{array}$ & $\begin{array}{c}\text { Basic } \\
\text { Events }\end{array}$ & $\begin{array}{c}\text { Repair Rates } \\
(\mathrm{R} / \mathrm{hr} .)\end{array}$ \\
\hline$\lambda_{A}$ & 0.001 & $\mu_{A}$ & 0.01 \\
$\lambda_{B}$ & 0.002 & $\mu_{B}$ & 0.01 \\
$\lambda_{C}$ & 0.003 & $\mu_{C}$ & 0.00 \\
\hline \hline
\end{tabular}

Repairable DFT is shown in Fig. 19 illustrates that in the case of finding the output of AND gate, events A and B can be repaired. In this system, it is assumed that event $\mathrm{C}$ is nonreparable. 


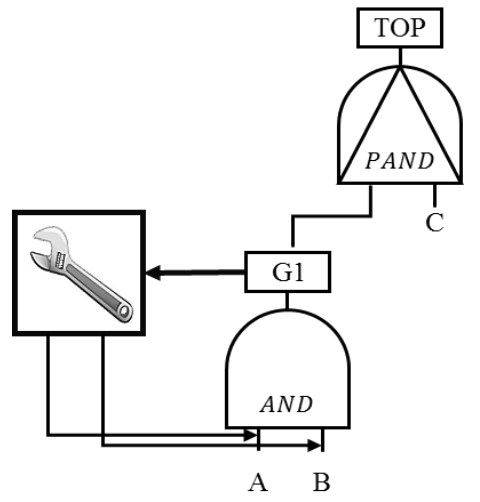

Fig. 19. A hypothetical repairable DFT with a repair gate.

The CTMC of this tree is illustrated in Fig. 20. It is assumed that the repair actions carry out immediately after failing the related events. This issue has been shown in the figure by dash transitions with repair rate $\mu$. In order to avoid model complexity in this figure, some self-transitions are not depicted.

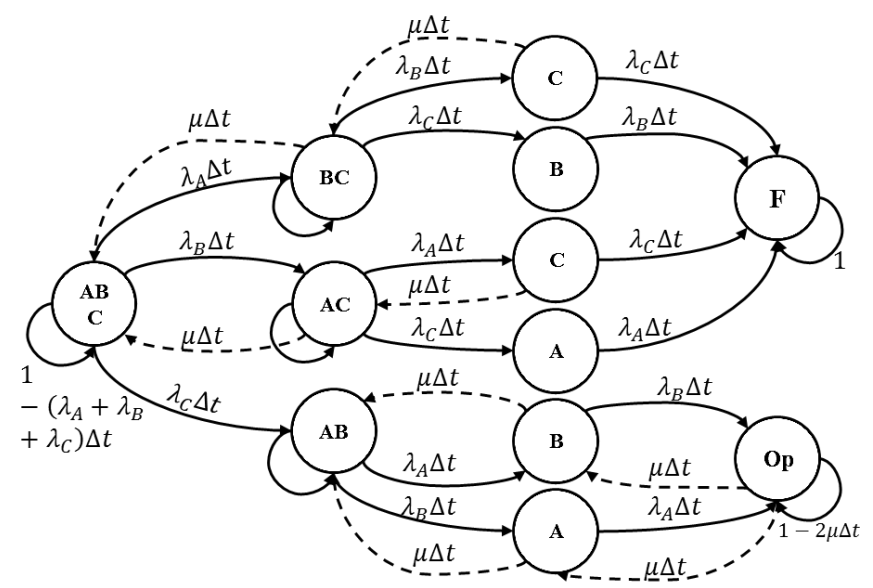

Fig. 20. CTMC of the repairable DFT of Fig. 19.

Recall that our hierarchical method solved the given DFT layer by layer in a bottom-top manner. For examples the DFT of Fig. 20 consists of two layers. First, the bottom layer (AND gate with repairable events; AND and repair gates) is solved. Its output is delivered to the left-hand-side input of PAND gate (up to a layer of the DFT). The semi-Markov model of this gate with an input for which the impact of repair has been considered is shown in Fig. 21. The dashed transitions defer the semi-Markov model of the PAND gate with repairable events from a simple PAND gate (a PAND gate with non-repairable events). The general equation of this gate is achieved through solving tits semi-Markov model by SMP theorem as follows. It should be noted that in this model $\mathrm{G}$ represents repair distribution function of the input's components which is equal to $G=1-e^{-\mu t}$, if repair distribution is considered exponential.

$$
R(t)=1-L^{-1}\left\{\frac{q_{1,2}(s) q_{2,4}(s)}{1-q_{1,2}(s) q_{2,1}(s)}\right\}
$$

where the $q_{i, j}(s)$ is the LST form of $Q_{i, j}(t)$ explained in section III.

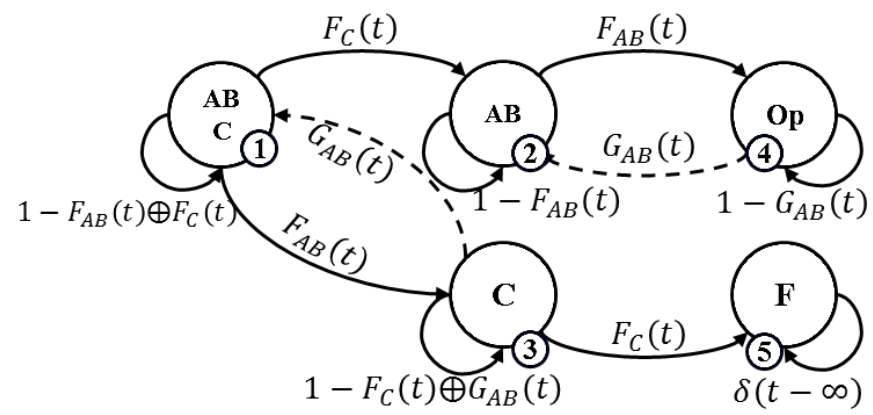

Fig. 21. Semi-Markov model for PAND gate with reparability in the first input

This procedure for solving DFT with repair gate (gates) can be applied for any other gates. This work is still under research by us.

Regarding both two presented models (CTMC and semiMarkov models), the unreliability behavior of the supposed DFT will be as Fig. 22 for 700 hours mission time. As seen from this figure, our method gives results very close to those achieved by the CTMC method. This verifies the correctness of our method.

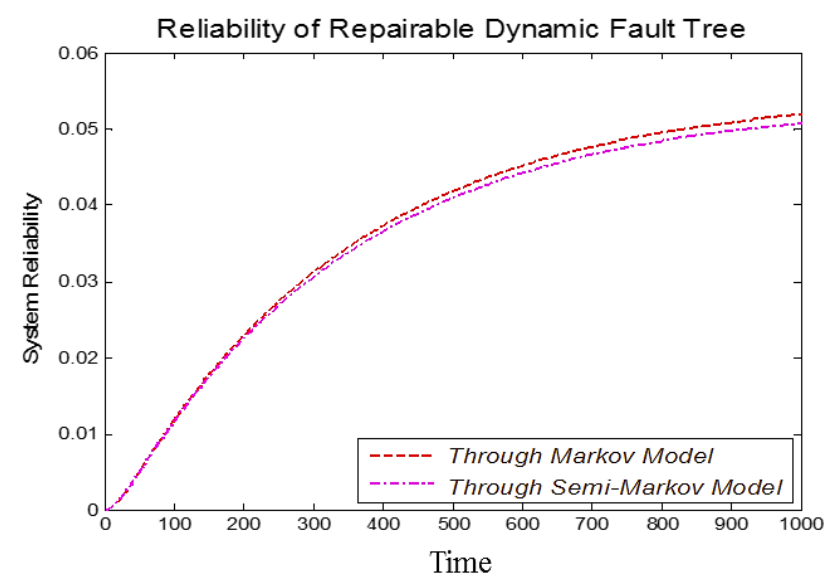

Fig. 22. The unreliability of repairable DFT in two states of solving by CTMC and solving by SMP theorem

\section{F. Example 6. Aircraft Fuel Distribution System}

As a case study, the Aircraft Fuel Distribution System (AFDS) has been chosen from [73] and its DFT from [74]. Fig. 23 illustrates the schematic of AFDS and Fig. 24 shows the DFT of the system that has been derived by HIP-HOPS tool [75, 74]. This system has two engines, seven bi-directional fuel pumps, five fuel tanks, and eleven valves enables the control system to choose active paths for fuel distribution in different conditions. The system also has six flow meters for fuel flow rate measurement. To refill the thanks, there is a refueling point and there are two jettison points for releasing the fuel in some situations. 


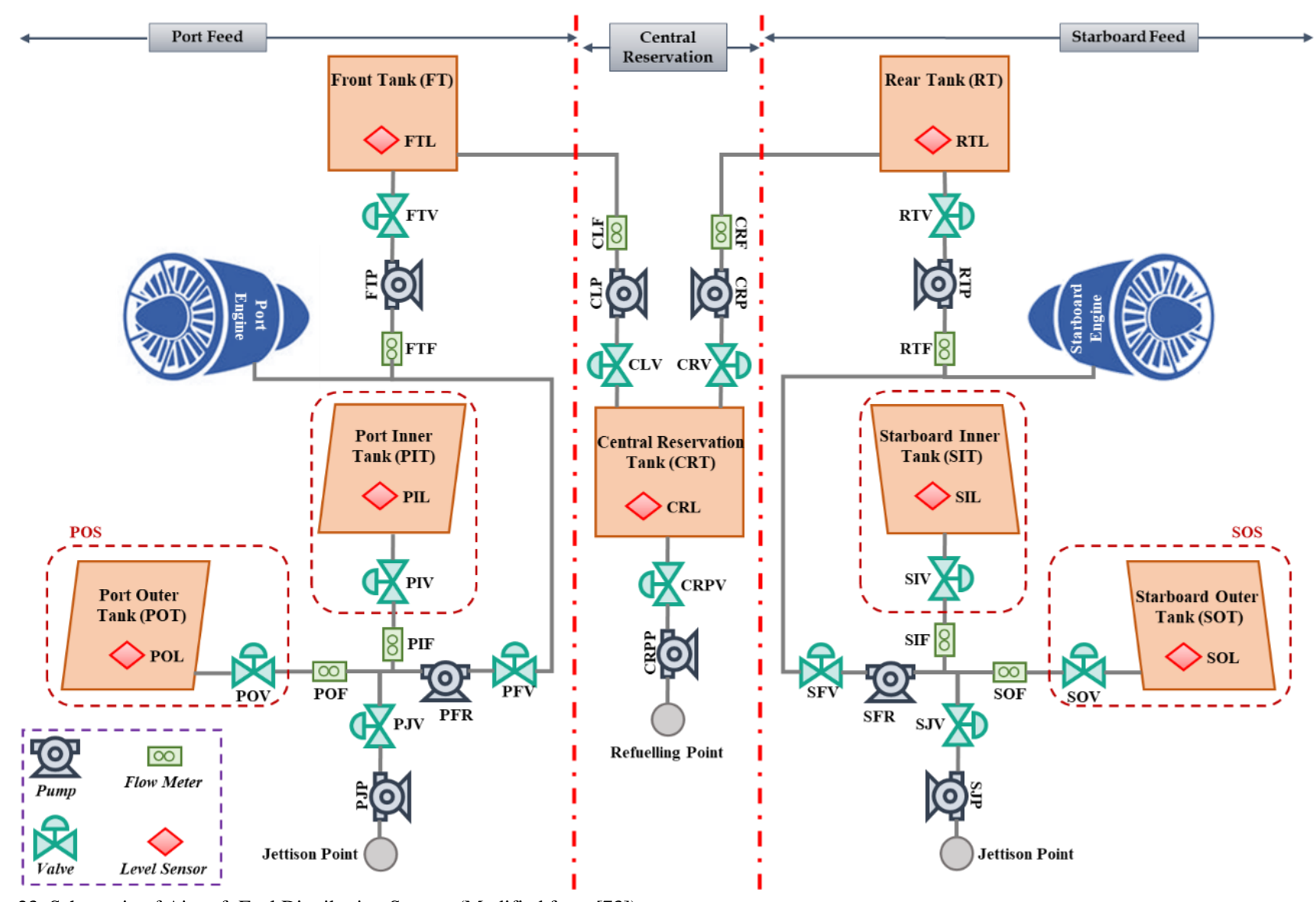

Fig. 23. Schematic of Aircraft Fuel Distribution System (Modified from [73])

The AFDS has been divided into three parts for performing the compositional analysis including Starboard Feed (SF), Central Reservation (CR) and Port Feed (PF) as delineated in Fig. 23. As can be seen, two SF and PF have identical components and each one of them has some subsystems. For instance, the SF includes Starboard Inner Subsystem (SIS), and Starboard Outer Subsystem (SOS). Through further decomposition of those subsystems, we have some components. For example, the SIS contains Starboard Inner fuel Level sensor (SIL), the valve (SIV) and Tank (SIT). Distribution of the fuel throughout the system and storing the fuel in the thanks are two main functions of AFDS and each function can be divided into two phases of refueling and consumption for different situations such as taxiing, take-off, cruising, approaching and landing. The fuel will be injected into Central Reservation Tank (CRT) in the refueling phase and then automatically distributed to Starboard and Port tanks. Moreover, the fuel will be consumed by both Starboard and Port engines in he consumption phase and certain level of fuel will be fed to engines. For more details regarding the ADFS please read [74]. In DFT of AFDS, It should be noted that "O- CompX" stands for omission of functionality of component X, "I-CompX" refers to the internal failure of component $\mathrm{X}$ and "Hi-CompX" includes erroneous high reading from component $\mathrm{X}$.

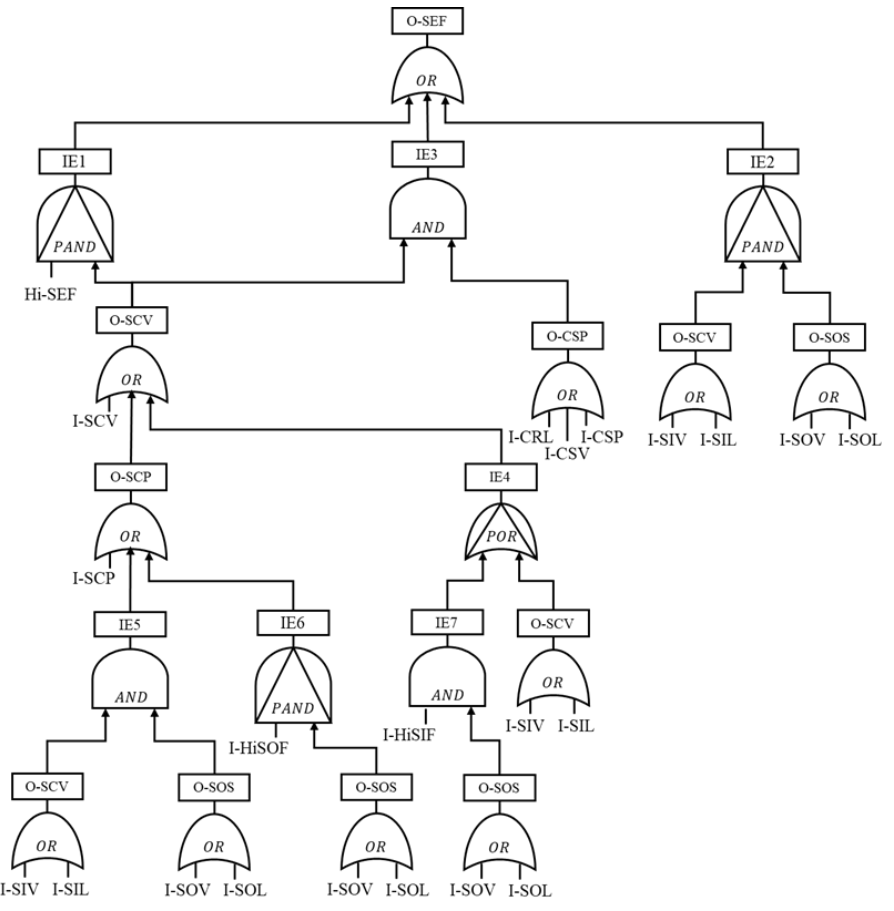

Fig. 24. Dynamic Fault Tree of Aircraft Fuel Distribution System [74]

The DFT of AFDS has twelve identical basic events (twenty two basic events counting the repeated events). Table VIII provides the failure rates and also short descriptions for the basic events. 
TABLE VIII

FAILURE RATES AND BASIC EVENTS IN DFT OF AFDS [74]

\begin{tabular}{|c|c|c|}
\hline Basic Events & Failure Rates (F/hr.) & Descriptions \\
\hline $\mathrm{I}-\mathrm{SCP}$ & $5.84267 \mathrm{E}-5$ & $\begin{array}{l}\text { Internal Failure of Starboard- } \\
\text { Central Pump }\end{array}$ \\
\hline I-CSP & $5.84267 \mathrm{E}-5$ & $\begin{array}{l}\text { Internal Failure of Central- } \\
\text { Starboard Pump }\end{array}$ \\
\hline I-SOV & $1.65633 \mathrm{E}-3$ & $\begin{array}{l}\text { Internal Failure of Starboard } \\
\text { Outer Valve }\end{array}$ \\
\hline I-SIV & $1.65633 \mathrm{E}-3$ & $\begin{array}{l}\text { Internal Failure of Starboard } \\
\text { Inner Valve }\end{array}$ \\
\hline $\mathrm{I}-\mathrm{CSV}$ & $1.65633 \mathrm{E}-3$ & $\begin{array}{l}\text { Internal Failure of Starboard } \\
\text { Central-Starboard Valve }\end{array}$ \\
\hline I-SCV & $1.65633 \mathrm{E}-3$ & $\begin{array}{l}\text { Internal Failure of Starboard } \\
\text { Starboard-Central Valve }\end{array}$ \\
\hline I-CRL & $2.21127 \mathrm{E}-6$ & $\begin{array}{l}\text { Internal Failure of Central } \\
\text { Reservation Level Sensor }\end{array}$ \\
\hline I-HiSOF & 4.06861E-5 & $\begin{array}{l}\text { Internal Failure or High Reading } \\
\text { From Starboard Outer Feed } \\
\text { Internal Failure or High }\end{array}$ \\
\hline I-HiSIF & $4.06861 \mathrm{E}-5$ & $\begin{array}{l}\text { Reading From Starboard Inner } \\
\text { Feed }\end{array}$ \\
\hline I-HiSEF & $4.06861 \mathrm{E}-5$ & $\begin{array}{l}\text { Internal Failure or High Reading } \\
\text { From Starboard Engine Feed }\end{array}$ \\
\hline I-SIL & $1.65633 \mathrm{E}-3$ & $\begin{array}{l}\text { Internal Failure of Starboard } \\
\text { Inner Level Sensor }\end{array}$ \\
\hline I-SOL & $3.31774 \mathrm{E}-5$ & $\begin{array}{l}\text { Internal Failure of Starboard } \\
\text { Outer Level Sensor }\end{array}$ \\
\hline
\end{tabular}

Reliability evaluation of AFDS through DFT has been addressed by [74]. They have converted the DFT to Petri Nets and Bayesian Networks and consequently calculated the reliability of the system. A comparison between the proposed approach and two other existing addressed methods has been provided in Table IX. The obtained results are approximate but close to the existing results.

TABLE IX

COMPARISON BETWEEN PROPOSED APPROACH, PETRI NETS BASED AND BAYESIAN NETWORKS BASED APPROACHES

\begin{tabular}{cccc}
\hline \hline $\begin{array}{c}\text { Mission } \\
\text { Time }\end{array}$ & $\begin{array}{c}\text { Proposed } \\
\text { Approach }\end{array}$ & Petri Nets [74] & $\begin{array}{c}\text { Bayesian } \\
\text { Networks [74] }\end{array}$ \\
\hline 100 & 0.05261416997 & 0.04998134610 & 0.04527486000 \\
500 & 0.59015071681 & 0.55645041000 & 0.52900833000 \\
1000 & 0.89056414810 & 0.87518982420 & 0.85004877000 \\
1500 & 0.96590195305 & 0.96217232090 & 0.94597227200 \\
2000 & 0.98766528020 & 0.98690630030 & 0.97782727200 \\
2500 & 0.99514980424 & 0.99502308600 & 0.99029561190 \\
3000 & 0.99801543278 & 0.99800983670 & 0.99563683150 \\
3500 & 0.99917346098 & 0.99918448590 & 0.99809093140 \\
4000 & 0.99965314139 & 0.99966205560 & 0.99916055205 \\
4500 & 0.99985398520 & 0.99985924100 & 0.99960976330 \\
5000 & 0.99993845915 & 0.99994123190 & 0.99980478790 \\
\hline \hline
\end{tabular}

Regarding the reliability evaluation of DFTs, there are some other challenges such as Common Cause Failures (CCF), and Reconfiguration that are not considered in this paper and can be studied as the future research.

\section{CAPABILITIES AND Limitations OF THE PROPOSED METHOD}

In this section, some of the capabilities and limitations (limitation in achieving an exact result) of the proposed method are discussed. A number of guidelines are suggested to overcome that limitation.

\section{A. Capabilities of the Proposed Method}

The proposed method offers the following capabilities:

1) This paper presents a universal semi-Markov model that can model any type of gates including static and dynamic. In addition, it embeds the functional dependencies behavior of gates' inputs into gate model. This simplifies the final model of DFT.

2) The proposed method solves DFTs hierarchically through SMP theorem. So, this method can reduce problem complexity in order to reduce state and transition explosion. Moreover, the presented general equations for gates help us to reduce SMP solution's trend.

3) The proposed method is able to consider non-exponential failure by means of SMP theorem. It is possible to consider hybrid failure distributions as the gate's input by the use of SMP theorem.

4) Basic events in this method can be defined by SMP or CTMC in which repair, imperfect coverage, and other issues can be considered. This idea already exists for static FT in the literature [76] and extended by us for DFTs.

\section{B. Limitations of the Proposed Method}

The proposed method has the following limitations. All will be resolved is our future works.

1) The results of this method are approximate for DFTs with repeated events make the results more approximate or less precise. It should be noted that the proximity of the method would not diminish its effectiveness since, in spite of the proximity, the results obtained through this method is much more precise than the results of some other published research works [29].

2) In the proposed method, the output of SEQ, OR, and POR gates with any complexity in their inputs will be precise. In addition, in the AND and PAND gates when a complex event or a sub-tree is connected to the first input and a simple event connected to the second input, the output will be precise. Otherwise, the output will be approximate for other gates and other situations for AND and PAND gates the output will be approximate.

3) In some benchmarks in which the shared or sliding spares are used, the proposed method is not able to solve shared spare gates. It is suggested to replace shared spares and use the reshaping rules (see appendix). It should be noted that these gates are then modelled as a semi-Markov model and generalized for any kind of failures distribution function.

4) In this study, it is assumed that the fault tree has only coherent events and there would be no guarantee for a fault tree with non-coherent events.

5) There are still some gates like pSAND and SAND that cannot be modelled through the universal gate. We hope to improve this universal gate to consider pSAND and more other gates as future research works. It would be also possible to define new gates such as semi-PAND in the future.

6) The example of repairable DFT is just provided to show that there might be a possibility to improve the approach for 
repairable DFTs. However, at the moment, there is no guarantee for any other example of repairable DFT. In fact, this example is just an insight into potential future works.

\section{CONCLUSION AND FUTURE WORK}

In this paper, a novel hierarchical approach to evaluating the reliability of DFTs based on SMP theorem was presented and the universal state space model has proposed for the static of dynamic gates (with inputs with exponential and nonexponential failure distribution function). The proposed method can compete with other approximate solutions for reliability evaluation of DFT. A number of examples have been given to show the capabilities and limitations of the proposed solution in I) parametric solution that can be used for other related computations such as MTTF and Sensitivity, II) dealing with non-exponential failure distribution functions, III) dealing with repeated basic events, IV) no state explosion V) considering repairable events (a limited example just to provide an insight for future research works) and VI) a case study of AFDS. Moreover, the limitations and capabilities of the proposed method have been discussed clearly.

SMP have limitations in modeling the concurrency among generally distributed events. Therefore, MRGPs and phasedapproximated (PH) approaches can be applied instead of SMP in the proposed method to use in a wider range of problems [2].

\section{APPENDIX}

The proposed method of this paper can solve DFTs with the shared spare gate by some reshape rules. We assume three types of shared CSP and reshaping them by LSH gate. LSH gate has four inputs, and their input is described by $F_{i}(t), i=1, \ldots, 4$. Fig. 25 shows how a given DFT with series shared CSP can be reshaped with LSH.
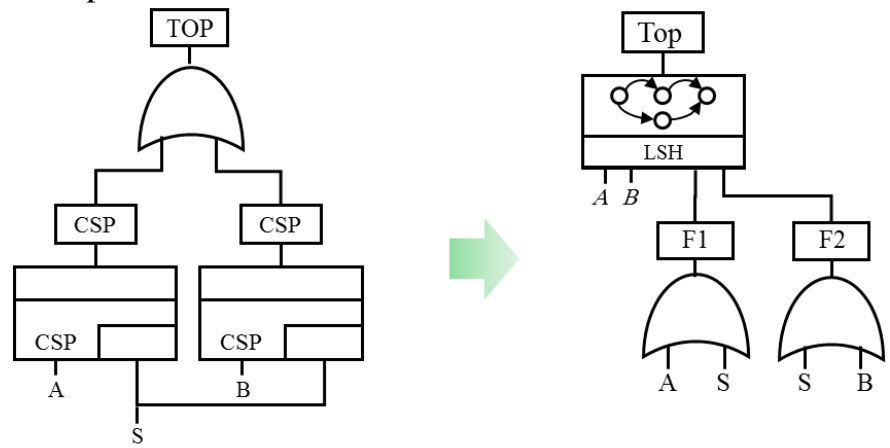

Fig. 25. Reshape series shared CSP's DFT by the use of LSH gate

Similar to the previous figure we can reshape the DFT of parallel shared CSP as Fig. 26. In addition, if we have priority in the parallel shared CSP system, then the DFT can be reshaped as Fig. 27.
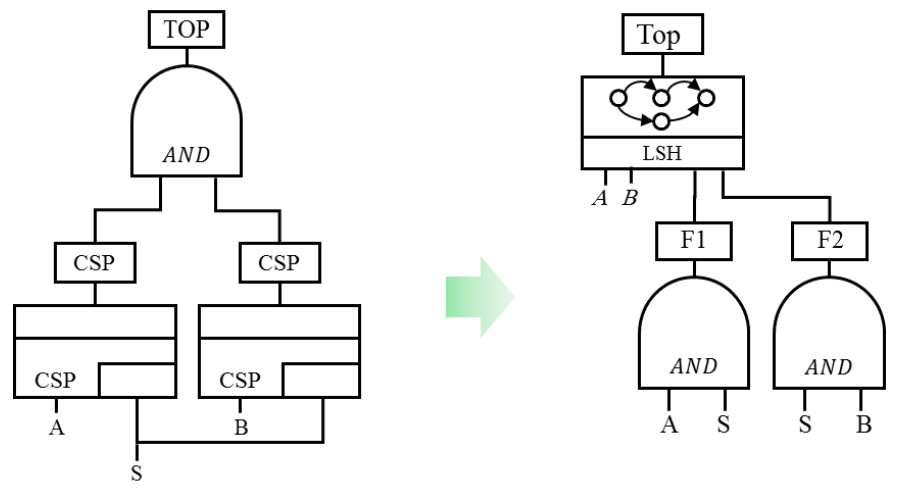

Fig. 26. Reshape parallel shared CSP's DFT by the use of LSH gate
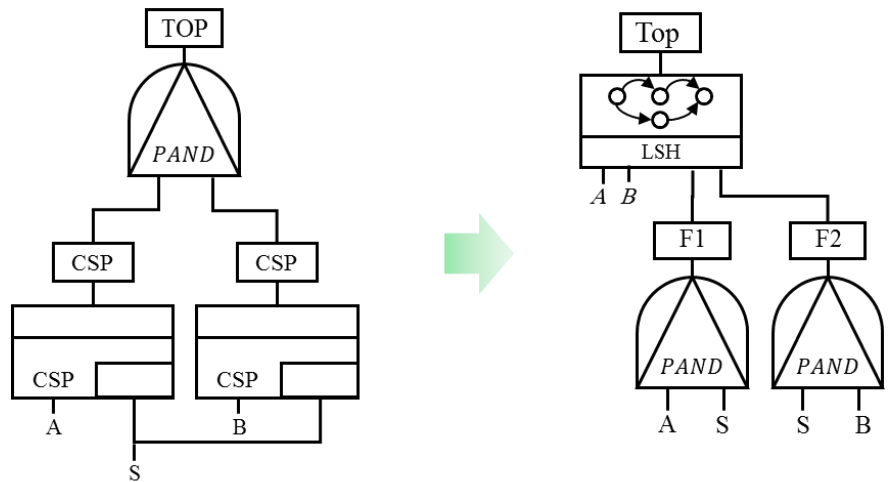

Fig. 27. Reshape DFT of parallel shared CSP with priority using LSH gate

\section{ACKNOWLEDGMENT}

We thank Mr. Mehrdad Mohammadi from Electronics and Computer Department, Shahid Beheshti University, Iran for his help that greatly improved the manuscript. We would like to show our gratitude to the Prof Yiannis Papadopoulos, Dr. Sohag Kabir and Dr. Youcef Gheraibia in the Dependable Intelligent Systems (DIS) Lab., University of Hull, for sharing their pearls of wisdom with us during the revision process of the paper, and we also thank three "anonymous" reviewers for their so-called insights.

\section{REFERENCES}

[1] E. Dubrova, Fault-Tolerant Design, New York Heidelberg Dordrecht London: Springer, 2012.

[2] S. Distefano, F. Longo and K. S. Trivedi, "Investigating Dynamic Reliability and Availability through State-Space Models," Computers \& Mathematics with Applications, vol. 64, no. 12, pp. 3701-3716, 2012.

[3] P. Zhu, J. Han, L. Liu and M. Zuo, "A Stochastic Approach for the Analysis of Fault Trees With Priority AND Gates," IEEE Transactions on Reliability, vol. 63, no. 2, pp. 480 - 494, 2014.

[4] G. Manno, F. Chiacchio, L. Compagno, D. D'Urso and N. Trapani, "MatCarloRe: An Integrated FT and Monte Carlo Simulink Tool for the Reliability Assessment of Dynamic Fault Tree," Expert Systems with Applications, vol. 39, no. 12, pp. 10334-10342., 2012.

[5] F. Chiacchio, M. Cacioppo, D. D'Urso, G. Manno, N. Trapani and L. Compagno, "A Weibull-based Compositional Approach for Hierarchical Dynamic Fault Trees," Reliability Engineering \& System Safety, vol. 109, no. 1, pp. 45-52, 2013. 
[6] L. Xing and S. V. Amari, "Fault Tree Analysis," in Handbook of performability engineering, Verlag London, Springer, 2008, pp. 595620 .

[7] D. S. Kim, R. Ghosh and K. S. Trivedi, "A Hierarchical Model for Reliability Analysis of Sensor Networks," in IEEE 16th Pacific Rim International Symposium on Dependable Computing, Tokyo, Japan, 2010.

[8] K. S. Trivedi, D. S. Kim and R. Ghosh, "System Availability Assessment Using Stochastic Models," Applied Stochastic Models in Business and Industry, vol. 29, no. 2, pp. 94-109, 2013.

[9] M. Zeller and F. Montrone, "Combination of Component Fault Trees and Markov Chains to Analyze Complex, Software-Controlled Systems," in IEEE 3rd International Conference on System Reliability and Safety (ICSRS), Barcelona, Spain, 2019.

[10] Y. Mo, "A Multiple-Valued Decision-Diagram-Based Approach to Solve Dynamic Fault Trees," IEEE Transactions on Reliability, vol. 63, no. 1, pp. $81-93,2014$.

[11] G. Merle, J. M. Roussel, J. Lesage and A. Bobbio, "Probabilistic Algebraic Analysis of Fault Trees with Priority Dynamic Gates and Repeated Events," IEEE Transactions on Reliability, vol. 59, no. 1, pp. 250-261, 2010.

[12] J. Ni, W. Tang and Y. Xing, "A Simple Algebra for Fault Tree Analysis of Static and Dynamic Systems," IEEE Transactions on Reliability, vol. 62, no. 4, pp. $846-861,2013$.

[13] G. Merle, J. M. Roussel and J. J. Lesage, "Algebraic Determination of the Structure Function of Dynamic Fault Trees," Reliability Engineering \& System Safety, vol. 96, no. 2, pp. 267-277, 2011.

[14] D. Codetta-Raiteri, "Extended Fault Trees Analysis Supported by Stochastic Petri Nets," PhD thesis, University of Torino, Torino, Italy, 2005.

[15] H. Boudali, P. Crouzen and M. Stoelinga, "A Rigorous, Compositional, and Extensible Framework for Dynamic Fault Tree Analysis," IEEE Transactions on Dependable and Secure Computing, vol. 7, no. 2, pp. 128-143, 2010.

[16] A. Ejlali and S. Ghassem Miremadi, "FPGA-based Monte Carlo Simulation for Fault Tree Analysis," Microelectronics Reliability, vol. 44, no. 6, pp. 1017-1028, 2004.

[17] J. Bechta Dugan, B. Venkataraman and R. Gulati, "DIFTree: A Software Package for the Analysis of Dynamic Fault Tree Models," in IEEE Reliability and Maintainability Symposium, Philadelphia, PA, 1997.

[18] E. Rothmann, J. B. Dugan, K. S. Trivedi, N. Mittal, M. A. Boyd, R. M. Geist and M. D. Smotherman, "HiRel: Hybrid Automated Reliability Predictor (HARP) Integrated Reliability Tool System (Version 7.0)," National Aeronautics and Space Administration (NASA), Langley Research Center, Hampton, VA, United States, 1994.

[19] B. Salvatore J, R. Elizabeth, D. Joanne Bechta, T. Kishor S, M. Nitin, B. Mark A and S. Mark D, "HiRel: Hybrid Automated Reliability Predictor (HARP) Integrated Reliability Tool System (Version 7.0) HARP Introduction and User"s Guide," National Aeronautics and Space Administration (NASA), Langley Research Center, Hampton, VA, United States, 2003.

[20] R. Manian, D. W. Coppit, K. J. Sullivan and J. B. Dugan, "Bridging the Gap Between Fault Tree Analysis Modeling Tools and the Systems Being $\quad 1999 . \quad$ [Online]. Available: http://citeseerx.ist.psu.edu/viewdoc/summary?doi=10.1.1.35.8466.

[21] M. A. Boyd, "Dynamic Fault Tree Models: Techniques for Analysis of Advanced Fault Tolerant Computer Systems," PhD Thesis, Department of Computer Science, Duke University, Durham, North Carolina, United States, 1991.

[22] J. Bechta Dugan, "Analysis of a Hardware and Software Fault Tolerant Processor for Critical Applications," in AIAA Computing in Aerospace, San Diego, Ca, 1993.

[23] S. A. Doyle, "Dependability Analysis of Fault Tolerant Systems: A New Look at Combinatorial Modeling," PhD Thesis, Department of Computer Science, Duke University, Durham, North Carolina, United Stetes, 1995.

[24] R. Manian, D. W. Coppit, K. J. Sullivan and J. Bechta Dugan, "Bridging the Gap Between Systems and Dynamic Fault Tree Models," in IEEE
Reliability and Maintainability Symposium (RAMS), Washington, DC, 1999.

[25] L. Yin, M. A. Smith and K. S. Trivedi, "Uncertainty Analysis in Reliability Modeling," in IEEE Reliability and Maintainability Symposium (RAMS), Philadelphia, PA, 2001.

[26] W. Vesely, J. Bechta Dugan, J. Fragola, J. Minarick and J. Railsback, "Fault Tree Handbook with Aerospace Applications," Office of Safety and Mission Assurance NASA Headquarters, Washington DC, 2002.

[27] C. Y. Huang and Y. R. Chang, "An Improved Decomposition Scheme for Assessing the Reliability of Embedded Systems by Using Dynamic Fault Trees," Reliability Engineering \& System Safety, vol. 92, no. 10, pp. 1403-1412, 2007.

[28] A. D. Domınguez-Garcıa, "An Integrated Methodology for the Performance and Reliability Evaluation of Fault-Tolerant Systems," PhD Thesis, Department of Electrical Engineering and Computer Science, Massachusetts Institute of Technology, United States, 2007.

[29] T. Yuge and S. Yanagi, "Quantitative Analysis of a Fault Tree with Priority AND Gates," Reliability Engineering \& System Safety, vol. 93, no. 11, pp. 1577-1583, 2008.

[30] T. Norberg, L. Rosén and A. Lindhe, "Added Value in Fault Tree Analyses," in Safety, Reliability and Risk Analysis: Theory, Methods and Applications, Valencia, Spain, 2009.

[31] A. K. Verma, A. Srividya and D. R. Karanki, "System Reliability Modeling," in Reliability and Safety Engineering, Verlag London, Springer, 2010, pp. 71-168.

[32] O. Yevkin, "An Improved Modular Approach for Dynamic Fault Tree Analysis," in IEEE Reliability and Maintainability Symposium (RAMS), Lake Buena Vista, FL, 2011.

[33] W. Han, W. Guo and Z. Hou, "Research on the Method of Dynamic Fault Tree Analysis.," in IEEE 9th International Conference on Reliability, Maintainability and Safety (ICRMS), Guiyang, China, 2011.

[34] S. Amari, G. Dill and E. Howald, "A New Approach to Solve Dynamic Fault Trees," in IEEE Reliability and Maintainability Symposium (RAMS), Tampa, FL, USA, 2003.

[35] W. G. Guo, W. Han and S. Y. Liu, "Dynamic Fault Tree Based on Weibull Distribution," Advanced Materials Research, vol. 308, no. 1, pp. 1322-1327, 2011.

[36] L. Zixian, N. Xin, L. Yiliu, S. Qinglu and W. Yukun, "Gastric Esophageal Surgery Risk Analysis With a Fault Tree and Markov Integrated Model," Reliability Engineering \& System Safety, vol. 96, no. 12, pp. 1591-1600, 2011.

[37] A. H. Ranjbar, M. Kiani and B. Fahimi, "Dynamic Markov Model for Reliability Evaluation of Power Electronic Systems," in IEEE International Conference on Power Engineering, Energy and Electrical Drives (POWERENG), Malaga, Spain, 2011.

[38] Y. F. Li, H. Z. Huang, Y. Liu and H. Li, "A New Fault Tree Analysis Method: Fuzzy Dynamic Fault Tree Analysis," Eksploatacja $i$ Niezawodnosc Maintenance and Reliability, vol. 14, no. 3, pp. 208-214, 2012.

[39] H. Hongzhong, L. Yanfeng, S. Jian, Y. Yuanjian and X. Ningcong, "Fuzzy Dynamic Fault Tree Analysis for the Solar Array Drive Assembly," Journal of Mechanical Engineering, 49(19), 19., vol. 49, no. 19, pp. 70-76, 2013.

[40] J. Xiang, F. Machida, K. Tadano, K. Yanoo, W. Sun and Y. Maeno, "A Static Analysis of Dynamic Fault Trees with Priority-AND Gates," in IEEE 6th Latin-American Symposium on Dependable Computing (LADC), Rio de Janeiro, 2013.

[41] J. Hao, L. Zhang and L. Wei, "Reliability Analysis Based on Improved Dynamic Fault Tree," in Engineering Asset Management, Verlag London, Springer, 2014, pp. 283-299.

[42] H. Hermanns, Interactive Markov Chains, Lecture Notes in Computer Science, Vol. 2428, Berlin: Springer, 2002.

[43] P. Crouzen, "Compositional Analysis of Dynamic Fault Trees using Input/Output Interactive Markov Chains," PhD Thesis, Faculty EWI, Computer Science Department, University Twente, Enschede, Netherlands, 2006.

[44] H. Boudali, P. Crouzen and M. Stoelinga, "Dynamic Fault Tree Analysis using Input/Output Interactive Markov Chains," in IEEE 37th 
International Conference on Dependable Systems and Networks, Edinburgh, Scotland, 2007.

[45] H. Boudali, P. Crouzen and M. Stoelinga, "A Compositional Semantics for Dynamic Fault Trees in Terms of Interactive Markov Chains," in Automated Technology for Verification and Analysis, Berlin Heidelberg, Springer, 2007, pp. 441-456.

[46] H. Boudali, P. Crouzen and M. Stoelinga, "Compositional Analysis of (Dynamic) Fault Trees (AFM7)," BRICKS: Basic Research in Informatics for Creating the Knowledge Society, Formal Methods \& Tools group, Faculty of EEMCS, University of Twente, Netherlands, 2008. [Online]. Available: http://www.bsikbricks.nl/research_posters_afm.shtml.

[47] F. Arnold, A. Belinfante, F. Van der Berg, D. Guck and M. Stoelinga, "DFTCalc: A Tool for Efficient Fault Tree Analysis," in Computer Safety, Reliability, and Security, Berlin Heidelberg, Springer, 2013, pp. 293-301.

[48] F. Arnold, A. Belinfante, F. Berg, D. Guck and M. Stoelinga, "DFTCalc: A Tool for Efficient Fault Tree Analysis (Extended Version)," Reported in University of Twente, Centre for Telematics and Information Technology (CTIT), Drienerlolaan, Netherlands, 2013.

[49] E. Ruijters and M. Stoelinga, "Fault Tree Analysis: A Survey of the State-of-the-art in Modeling, Analysis and Tools," Computer Science Review, vol. 15, no. 1, pp. 29-62, 2015.

[50] D. Ge and Y. Yang, "Reliability Analysis of Non-repairable Systems Modeled by Dynamic Fault Trees with Priority AND Gates," Applied Stochastic Models in Business and Industry, vol. 31, no. 6, pp. 809-822, 2015.

[51] P. A. Brameret, A. Rauzy and J. M. Roussel, "Automated Generation of Partial Markov Chain From High Level Descriptions," Reliability Engineering \& System Safety, vol. 139, no. 1, pp. 179-187, 2015.

[52] O. Yevkin, "An Efficient Approximate Markov Chain Method in Dynamic Fault Tree Analysis," Quality and Reliability Engineering International, vol. 32, no. 4, pp. 1509-1520, 2016.

[53] G. Manno, F. Chiacchio, L. Compagno, D. D'Urso and N. Trapani, "Conception of Repairable Dynamic Fault Trees and Resolution by The Use of RAATSS, A Matlab® Toolbox Based on the ATS Formalism," Reliability Engineering \& System Safety, vol. 121, no. 1, pp. 250-262, 2014.

[54] S. Junges, D. Guck, J. P. Katoen and M. Stoelinga, "Uncovering Dynamic Fault Trees," in 46th Annual IEEE/IFIP International Conference on Dependable Systems and Networks (DSN), Toulouse, France, 2016.

[55] Z. Ramezani, G. R. Latif-Shabgahi, P. Khajeie and K. Aslansefat, "Hierarchical Steady-State Availability Evaluation of Dynamic Fault Trees through Equal Markov Model," in IEEE 24th Iranian Conference on Electrical Engineering (ICEE), Tehran. Iran, 2016.

[56] F. Chiacchio, J. I. Aizpurua, D. D'Urso and L. Compagno, "Coherence Region of the Priority-AND Gate: Analytical and Numerical Examples," Quality and Reliability Engineering International, vol. 34, no. 1, pp. 107115, 2018.

[57] D. Ge and Y. Yang, "Negating a generalized cut sequence: Bridging The Gap Between Dynamic Fault Trees Quantification and Sum of Disjoint Products Methods," Quality and Reliability Engineering International, vol. 33, no. 2, pp. 357-367, 2017.

[58] P. Y. Piriou, J. M. Faure and J. J. Lesage, "Generalized Boolean logic Driven Markov Processes: A Powerful Modeling Framework for ModelBased Safety Analysis of Dynamic Repairable and Reconfigurable Systems," Reliability Engineering \& System Safety, vol. 163, no. 1, pp. 57-68, 2017.

[59] M. Volk, S. Junges and J. P. Katoen, "Fast Dynamic Fault Tree Analysis by Model Checking Techniques," IEEE Transactions on Industrial Informatics, vol. 14, no. 1, pp. 370-379, 2018.

[60] M. Zajac and A. Kierzkowski, "Attempts at Calculating Chosen Contributors with Regard to the Semi-Markov Process and the Weibull Function Distribution," in Summer Safety and Reliability Seminars (SSARS), Gdañsk-Sopot, Poland, 2011.

[61] V. G. Kulkarni, Modelling and Analysis of Stochastic Systems, London, UK: Chapman \& Hall, 1995.
[62] L. Yin, R. M. Fricks and K. Trivedi, "Application of Semi-Markov Process and CTMC to Evaluation of UPS System Availability," in Annual Reliability and Maintainability Symposium, Seattle, WA, 2002.

[63] F. Frattini, A. Bovenzi, J. Alonso and K. Trivedi, "Reliability Indices," in Wiley Encyclopedia of Operations Research and Management Science, John Wiley \& Sons, Inc., Hoboken, NJ, 2013, pp. 1-21.

[64] R. Fricks, M. Telek, A. Puliafito and K. Trivedi, "Markov Renewal Theory Applied to Performability Evaluation," in State-of-the-art in Performance Modeling and Simulation. Modeling and Simulation of Advanced Computer Systems: Applications and Systems, Newark, NJ, Gordon \& Breach, 1998, pp. 193-236.

[65] D. Rios Insua, F. Ruggeri and M. P. Wiper, "Appendix B: Generating Functions and the Laplace-Stieltjes Transform," in Bayesian Analysis of Stochastic Process Models, Chichester, UK. doi: 10.1002/9780470975916.app2, John Wiley \& Sons, Ltd, 2012, pp. 283 284.

[66] M. Zajac and T. Budny, "On Determination of Some Characteristics of Semi-Markov Process for Different Distributions of Transient Probabilities," Journal of Reliability \& Risk Analysis, vol. 2, no. 1, pp. 74-84, 2009.

[67] K. Aslansefat, "A Novel Approach for Reliability and Safety Evaluation of Control Systems with Dynamic Fault Tree," MSc. Thsis, Abbaspur Campus, Shahid Beheshti University, Tehran, Iran, 2014.

[68] L. Xing, P. Boddu and Y. \& Sun, "System Reliability Analysis Considering Fatal and Non-Fatal Shocks in a Fault Tolerant System," in IEEE Reliability and Maintainability Symposium, Fort Worth, TX, 2009.

[69] H. Boudali and J. Bechta Dugan, "A Discrete-Time Bayesian Network Reliability Modeling and Analysis Framework," Reliability Engineering and System Safety, vol. 87, no. 3, p. 337-349, 2005.

[70] G. Ciardo, R. A. Marie, B. Sericola and K. S. Trivedi, "Performability Analysis Using Semi-Markov Reward Processes.," IEEE Transactions on Computers, vol. 39, no. 10, pp. 1251-1264, 1990.

[71] D. Codetta-Raiteri, "Integrating Several Formalisms in Order to Increase Fault Trees' Modeling Power," Reliability Engineering \& System Safety, vol. 96, no. 5, pp. 534-544, 2011.

[72] L. Portinale, D. Raiteri and S. Montani, "Supporting Reliability Engineers in Exploiting the Power of Dynamic Bayesian Networks," International journal of approximate reasoning, vol. 51, no. 2, pp. 179195, 2010.

[73] E. Edifor, N. Gordon, M. Walker and Y. Papadopoulos, "Using Simulation to Evaluate Dynamic Systems with Weibull or Lognormal Distributions," in Proceedings of the Ninth International Conference on Dependability and Complex Systems DepCoS-RELCOMEX, Brunów, Poland, 2014

[74] S. Kabir, M. Walker and Y. Papadopoulos, "Dynamic System Safety Analysis in HiP-HOPS with Petri Nets and Bayesian Networks," Safety Science, vol. 105, no. 1, pp. 55-70, 2018.

[75] Y. Papadopoulos, J. Mcdermid, R. Sasse and G. Heiner, "Analysis and Synthesis of the Behaviour of Complex Programmable Electronic Systems in Conditions of Failure," Journal of Reliability Engineering and Sytem Safety, vol. 71, no. 1, p. 229-247, 2001.

[76] D. S. Kim, R. Ghosh and K. S. Trivedi, "A Hierarchical Model for Reliability Analysis of Sensor Networks," in IEEE 16th Pacific Rim International Symposium on Dependable Computing (PRDC), Tokyo, Japan, 2010. 


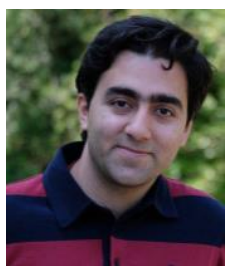

Koorosh Aslansefat was born in Tehran, Iran, in 1989. He received a $\mathrm{BSc}$ in Marine Electronic and Communication Engineering from Chabahar Maritime University (CMU), Chabahar, Iran in 2011 and ranked 1st among BSc students of Electronic department. He got MSc in Control Engineering at Shahid Beheshti University, Tehran, Iran in 2014. He is a Ph.D. student at the University of Hull. His main research interests are in Markov Modelling, Performance Assessment, Artificial Intelligent, Optimization, and Stochastic Modelling.
Gholamreza Latif-Shabgahi is an Assistant Professor in the Control Engineering Department at Shahid Beheshti University. He

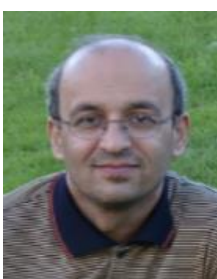
received his BSc. Degree in Electrical Engineering from Amirkabir University, Tehran, Iran. For his PhD and Postdoc degree in Control and Real Time Control Engineering, he went to Sheffield and Wales-Bangor Universities, respectively in United Kingdom. $\mathrm{He}$ is interested in Fault Tolerant Control, Sensor Networks and Reliable Systems, and published many research papers and articles in international conferences and journals. 